\title{
NEURAL NETWORKS IN FINANCE: A DESCRIPTIVE SYSTEMATIC REVIEW
}

\author{
Dr. K. Riyazahmed \\ Assistant Professor \\ Shri Dharmasthala Manjunatheshwara \\ Institute for Management Development \\ Mysore, Karnataka, India \\ E-mail: riyazahmed@sdmimd.ac.in
}

\begin{abstract}
Traditional statistical methods pose challenges in data analysis due to irregularity in the financial data. To improve accuracy, financial researchers use machine learning architectures for the past two decades. Neural Networks $(N N)$ are a widely used architecture in financial research. Despite the wider usage, NN application in finance is yet to be well defined. Hence, this descriptive study classifies and examines the NN application in finance into four broad categories i.e., investment prediction, credit evaluation, financial distress, and other financial applications. Likewise, the review classifies the NN methods used under each category into standard, optimized and hybrid NN. Further, accuracy measures used by the research work widely differ, in turn, pose challenges for comparison of a NN under each category and reduces the scope of formalizing a theory to choose optimum network model under each category.
\end{abstract}

Keywords: Neural Networks, ANN, Analytics, Machine Learning.

JEL Classification Codes: G1, G17, M150.

\section{INTRODUCTION}

Financial data are immensely available, yet the innate nature of big data shows uncertainty, incompleteness, and inconsistency which pose challenges in using traditional statistical methods for financial data analysis (Brooks et al., 2019; Hariri et al., 2019). Financial researchers try to overcome the traditional statistical limitations by using machine learning architecture like Neural Networks (NN).

NN imitates the human brain by using nodes and layers of connections, which pass signals with a set of associated weights and bias adjustments (Figure 1). NN results are not easily interpretable and so the analysis is in black-box nature. Irrespective of the non-interpretability of results, $\mathrm{NN}$ received importance in financial research due to its computing efficiency in handling financial big data.

For the last two decades, financial researchers are using $\mathrm{NN}$ in various analyses like risk classification (Altman, Marco, \& Varetto, 1994), bankruptcy and share price prediction (Barr \& Mani, 1994). Despite the attention, NN application in finance is yet to be well defined. The last decade saw an increase in the finance research using $\mathrm{NN}$ in an analysis like forecasting of share prices (Chang, Liu, Fan, Lin, \& Lai, 2009; Sapna \& Argente, 2003; Safer, 2002), option prices 
(Lin \& Yeh, 2009; Kohler, Krzyzak, \& Todorovic, 2010), and the future prices (Dunis, Laws, \& Evans, 2008; Laws \& Dunis, 2013).

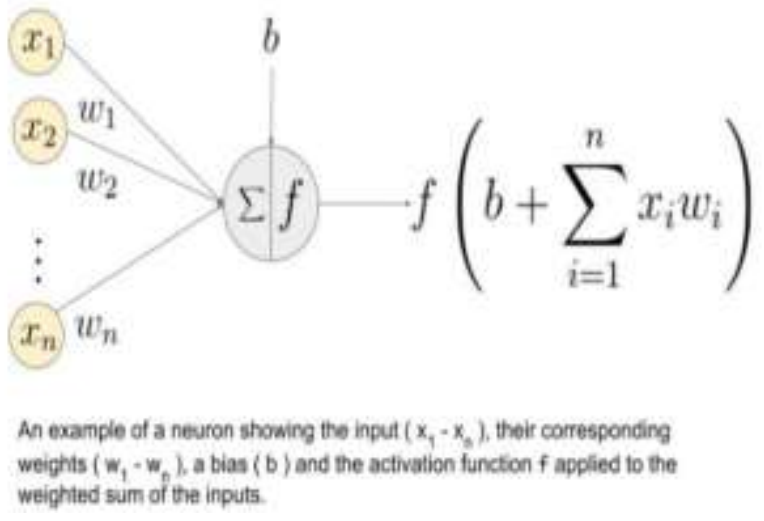

Figure 1. standard Neural Network

The Literature reviews of Feldman and Kingdon (1995), Wong and Selvi (1998), Vellido, Lisboa, and Vaughan (1999), Krishnaswamy, Gilbert, and Pashley (2000), Coakley and Brown (2000), Fadlalla and Lin (2001), Wei, Nakamori, Wang, and Yu (2007), Cavalcante et al. (2016), and Huang, Chai, and Cho (2020) are the existing works in this regard. Yet, the review works show significant limitations. Firstly, no review follows a protocol-based review process which is essential for reproducibility. Secondly, several reviews are not examining financial applications entirely or they focus on aspects like soft computing, and computational intelligence, instead of NN architectures (Table 1). Even though the studies analyze the NN applications to a certain extent, non-reproducibility is a serious concern.

Further, the absence of a systematic review method results in serious drawbacks in the quality of review findings (Karunananthan, Maxwell, \& Welch, 2020). Systematic reviews have greater potential than other research designs leading to the reproducibility of research (Shokraneh \& Adams, 2019). Since the computing efficiency doubles every two years (Gustafson, 2011) which improves the efficiency of handling complex data sets, exploring the research works to date with the scientific review methods will help to understand the existing status of NN in analyzing the financial data.

Table 1. Summary of existing reviews

\begin{tabular}{|l|l|l|l|l|}
\hline Study & $\begin{array}{l}\text { Period of } \\
\text { review }\end{array}$ & $\begin{array}{l}\text { Nature of } \\
\text { study }\end{array}$ & $\begin{array}{l}\text { Focus of the } \\
\text { study }\end{array}$ & Summary of conclusion \\
\hline $\begin{array}{l}\text { Feldman and } \\
\text { Kingdon (1995) } \\
\text { (*Authors, } \\
\text { estimation) }\end{array}$ & $\begin{array}{l}\text { Descriptive, } \\
\text { Non- } \\
\text { Systematic } \\
\text { review. }\end{array}$ & $\begin{array}{l}\text { Advantages of } \\
\text { MLP, BPN, } \\
\text { and SOM. }\end{array}$ & $\begin{array}{l}\text { Generalization, } \\
\text { architecture selection, } \\
\text { and application of } \\
\text { selected NN. }\end{array}$ \\
\hline $\begin{array}{l}\text { Wong and Selvi } \\
(1998)\end{array}$ & $1990-1996$ & $\begin{array}{l}\text { Descriptive, } \\
\text { Review } \\
\text { process } \\
\text { disclosed. }\end{array}$ & $\begin{array}{l}\text { Classification } \\
\text { of NN } \\
\text { application in } \\
\text { finance. }\end{array}$ & $\begin{array}{l}\text { The implication to NN } \\
\text { developers. }\end{array}$ \\
\hline Velido, Lisboa, & $1992-1998$ & Descriptive, & Application of & Comprehensively \\
\hline
\end{tabular}




\begin{tabular}{|c|c|c|c|c|}
\hline $\begin{array}{ll}\text { and } & \text { Vaughan } \\
(1999) & \end{array}$ & & $\begin{array}{l}\text { Review } \\
\text { process } \\
\text { disclosed. }\end{array}$ & $\begin{array}{l}\mathrm{NN} \\
\text { business. }\end{array}$ & $\begin{array}{l}\text { reported the most quoted } \\
\text { advantages and } \\
\text { disadvantages of NN in } \\
\text { various business } \\
\text { applications. }\end{array}$ \\
\hline $\begin{array}{l}\text { Krishnaswamy et } \\
\text { al. (2000) }\end{array}$ & $\begin{array}{l}1989-1996 \\
\text { (*Authors' } \\
\text { estimation) }\end{array}$ & $\begin{array}{l}\text { Descriptive, } \\
\text { Non } \\
\text { systematic } \\
\text { review }\end{array}$ & $\begin{array}{l}\text { Description of } \\
\mathrm{NN} \text { and its } \\
\text { finance } \\
\text { application. }\end{array}$ & $\begin{array}{l}\text { Backpropagation } \mathrm{NN} \text { has } \\
\text { proven robust. } \\
\text { Supervised and } \\
\text { unsupervised NN is used } \\
\text { in finance. }\end{array}$ \\
\hline $\begin{array}{l}\text { Coakley and } \\
\text { Brown (2000) }\end{array}$ & $\begin{array}{l}1988-1997 \\
\text { (*Authors' } \\
\text { estimation) }\end{array}$ & $\begin{array}{l}\text { Descriptive, } \\
\text { Non } \\
\text { systematic } \\
\text { review }\end{array}$ & $\begin{array}{l}\text { Financial } \\
\text { Application, } \\
\text { development } \\
\text { of ANN } \\
\text { models. }\end{array}$ & $\begin{array}{l}\text { ANN researchers face a } \\
\text { challenge that there are } \\
\text { no formal theories for } \\
\text { determining optimal } \\
\text { network model }\end{array}$ \\
\hline $\begin{array}{l}\text { Fadlalla and Lin } \\
\text { (2001) }\end{array}$ & $1986-1997$ & $\begin{array}{l}\text { Descriptive, } \\
\text { Non } \\
\text { systematic } \\
\text { review }\end{array}$ & $\begin{array}{l}\text { Financial } \\
\text { Application, } \\
\text { focus on } \\
\text { feedforward } \\
\text { and feed } \\
\text { backward NN } \\
\text { models. }\end{array}$ & $\begin{array}{l}\text { NN has great promise } \\
\text { for financial applications } \\
\text { and combinations of two } \\
\text { approaches should be } \\
\text { investigated. }\end{array}$ \\
\hline $\begin{array}{ll}\text { Calderon } & \text { and } \\
\text { Cheh }(2002) & \end{array}$ & $1993-1999$ & $\begin{array}{l}\text { Descriptive, } \\
\text { sources of } \\
\text { review } \\
\text { disclosed. }\end{array}$ & $\begin{array}{l}\mathrm{NN} \text { in } \\
\text { auditing and } \\
\text { risk } \\
\text { management. }\end{array}$ & $\begin{array}{lr}\text { NN shows promising } \\
\text { performance } \\
\text { Preliminary analytical } \\
\text { procedures in the } \\
\text { auditing process. }\end{array}$ \\
\hline $\begin{array}{l}\text { Wei Huang et al. } \\
(2007)\end{array}$ & NA & $\begin{array}{l}\text { Descriptive, } \\
\text { Non } \\
\text { systematic } \\
\text { review }\end{array}$ & $\begin{array}{l}\text { Focus on input } \\
\text { variables, NN } \\
\text { models } \\
\text { applied in } \\
\text { forex, stock } \\
\text { market, and } \\
\text { economic } \\
\text { forecasting. }\end{array}$ & \begin{tabular}{lrr} 
The & \multicolumn{2}{c}{ prediction } \\
performance of neural \\
networks can \\
improved be by being \\
integrated with other \\
technologies.
\end{tabular} \\
\hline $\begin{array}{l}\text { Cavalcante et al. } \\
(2016)\end{array}$ & $2009-2015$ & $\begin{array}{l}\text { Descriptive, } \\
\text { review process } \\
\text { disclosed }\end{array}$ & $\begin{array}{l}\text { Computational } \\
\text { intelligence in } \\
\text { finance (NN is } \\
\text { a part of the } \\
\text { study) }\end{array}$ & $\begin{array}{l}\text { Categorized studies into } \\
\text { preprocessing, } \\
\text { forecasting, and text } \\
\text { mining. }\end{array}$ \\
\hline $\begin{array}{l}\text { Huang et al. } \\
(2020)\end{array}$ & $2014-2018$ & $\begin{array}{l}\text { Descriptive, } \\
\text { review } \\
\text { collection } \\
\text { process } \\
\text { disclosed. }\end{array}$ & $\begin{array}{l}\text { Deep learning } \\
\text { applications in } \\
\text { finance and } \\
\text { banking. }\end{array}$ & $\begin{array}{l}\text { Reports about data } \\
\text { inputs, preprocessing } \\
\text { and evaluation rules of } \\
\text { deep learning in finance } \\
\text { and banking }\end{array}$ \\
\hline
\end{tabular}


Hence, this structured review reveals an interpretable pattern of NN architectures and their application in finance research. The study follows the systematic review process provided by Moher, Liberati, Tetzlaff, Altman, and The PRISMA Group (2009) and Gupta, Chauhan, and Jaiswal (2019). The primary aim of the study is to classify the research papers based on their NN application in finance research. This is done by,

- Classification of major topics and sub-topics, and

- Identification of various NN architectures used in the classified subtopics.

\section{METHOD}

A literature review starts with searching for quality research papers from prominent journals (Ngai \& Wat, 2002). Further collecting research papers from the online database has become an emerging culture in the information era (Petter \& Lean 2009). So, this study uses the EBSCO Business Elite database, which is a repository of 525 peer-reviewed research journals, to collect research papers. The systematic review process prescribed by Moher et al. (2009) comprises defining a protocol for literature search, exclusion criteria of research papers, and final selection of papers (Figure 2).

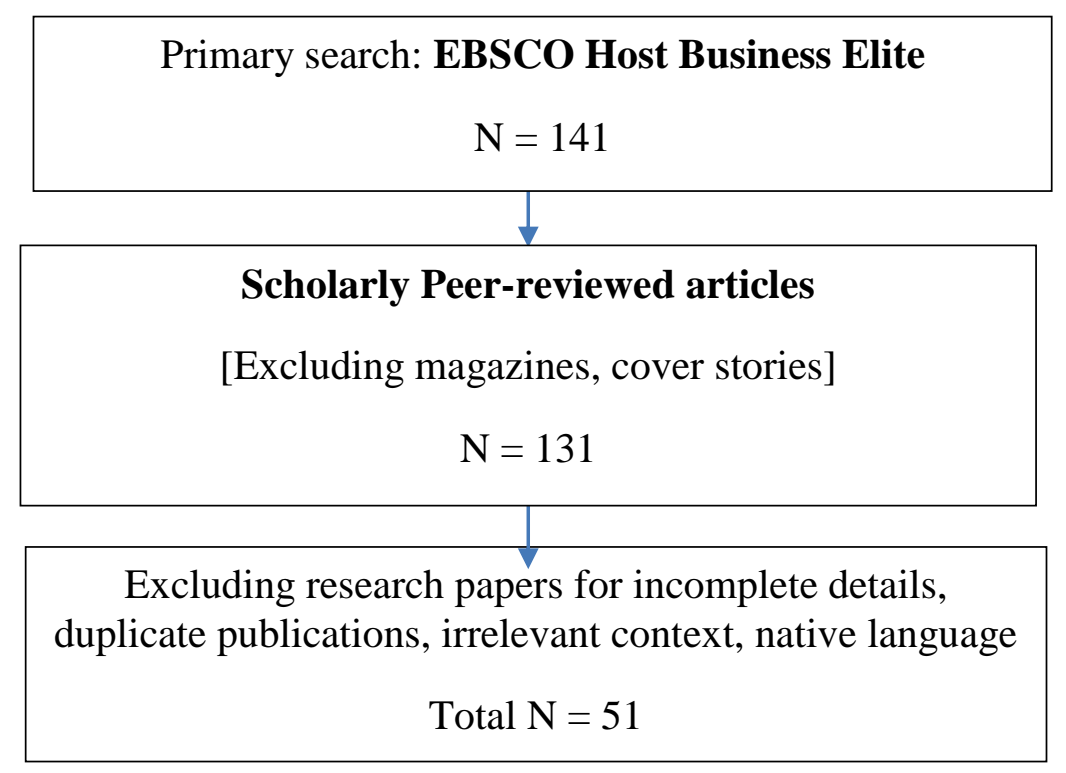

Figure 2. Selection process of research papers (Moher et al., 2009)

Protocol directs the research paper selection based on the criteria. To obtain research papers the study followed an advanced search option in the EBSCO Business Elite database and used two keywords 'Neural Networks' and 'Finance'. Research studies published in English under the subject areas of business, management, and finance are only considered. Empirical articles that are published in peer-reviewed academic journals are collected at the first level.

Restricting the review only to published articles can strengthen quality control since many of the academic journals follow meticulous publishing criteria in terms of research contribution and robustness of the results (Light \& Pillemer, 1984). The protocol process has helped to collect papers with high research quality. After collection, the studies with incomplete details, irrelevant context, and duplicate publications are excluded. 
After exclusions, data extraction is done by carefully considering the title, abstract, and overall theme of the paper focuses on applying $\mathrm{NN}$ in finance. 141 papers are collected from the EBSCO Business Elite database and during the first level of screening 77 research papers are excluded. Finally, 51 research papers are considered for review.

\section{RESULTS}

The researcher scrutinized the collected research articles for their relevancy and suitability to be considered as a part of this review paper. When research papers fulfill the established criteria, the author read the full paper to find its contributions. Figure 3 represents the broader classification of research papers under major topics and sub-topics.

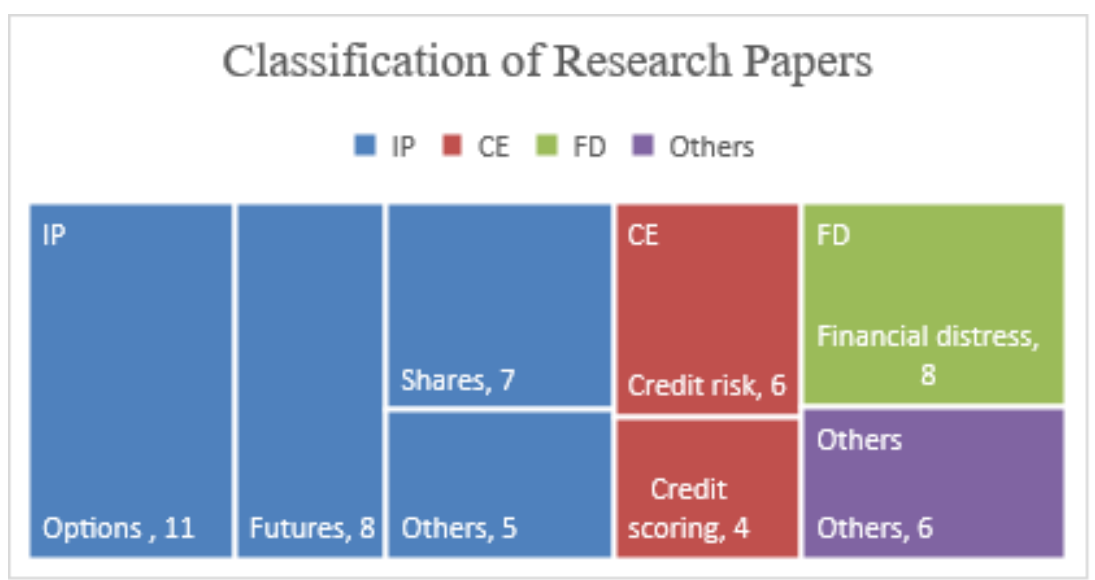

IP - Investment Prediction; CE - Credit Evaluation; FD - Financial Distress.

Figure 3. Classification of research papers based on major topics and sub-topics

Based on the reading the author identified four main topics and several subtopics (Table 2). The next section discusses each main topic, inferences of the research work carried in the subtopics based on the NN methods. Further, NN with statistical and architectural advancements is classified as 'Optimized NN'. NNs incorporating financial theories and knowledge are classified under 'Hybrid NN'. A model-free NN is classified as 'Standard NN'.

Table 2. Major research topics and subtopics

\begin{tabular}{|l|l|}
\hline Main topics & Sub-topics \\
\hline Investment prediction & $\begin{array}{l}\text { Prediction of options prices, futures prices, share prices, Forex, } \\
\text { indexes, bond yields, commodity spreads, trading patterns, } \\
\text { shareholder wealth, and portfolio performance. }\end{array}$ \\
\hline Credit evaluation & $\begin{array}{l}\text { Predicting credit risk, and } \\
\text { Estimating credit rating. }\end{array}$ \\
\hline Financial distress & Evaluating financial distress \\
\hline Other financial applications & $\begin{array}{l}\text { Development of financial intelligent system, } \\
\text { Detecting fraudulent financial reporting, } \\
\text { Assessment of systematic risk, } \\
\text { Evaluating operating performance, } \\
\text { Assessing project portfolio performance. }\end{array}$ \\
\hline
\end{tabular}




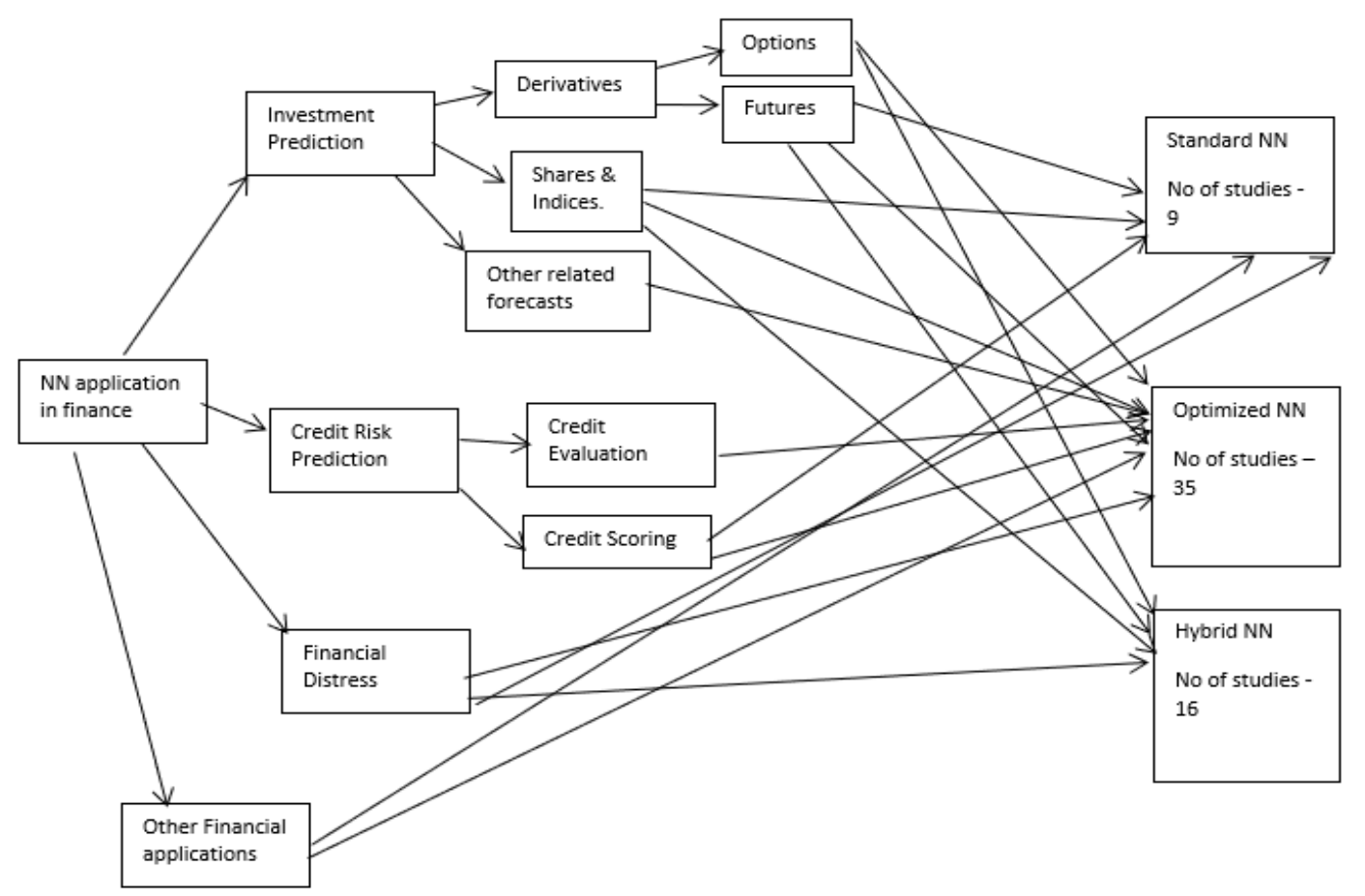

Figure 4. Framework of NN application in finance

\section{DISCUSSION}

\section{Investment Prediction:}

\section{Derivatives -}

\section{a) Options}

\section{Optimized NN -}

Back Propagation NN (BPNN) - BPNN minimizes the prediction error by giving the nodes with higher error rates lower weights and vice versa. Hence, BPNN is found to be more suitable for derivative prediction (Kaastra \& Boyd, 1995). When used to predict Taiwan stock index options, BPNN demonstrated improved accuracy in support of hedging at in-the-money option (Lin \& Yeh, 2009).

Likewise, the research study of Hutchison et al. (1994) used BPNN to predict the prices of S \& P 500 futures and options, yet the result is that BPNN did not show significantly better accuracy than other linear models like ordinary least squares.

NN with Monte Carlo simulation - Monte Carlo, is a simulation technique to understand the impact of risk and uncertainty in prediction. It is used with linear NN for predicting American options (Kohler et al., 2010). Since it is a simulation based NN, the accuracy would widely differ in empirical prediction.

Advanced Modular NN (AMNN) - AMNN is a series of independent NN which serves as a module and operates on separate inputs to accomplish a subtask. AMNN gives more accuracy than the standard NN when predicting European call option prices (Gradojevic et al., 2009). 
Feed forward NN (FFNN) - FFNN is used when the nature of financial data is neither sequential nor time-dependent. FFNN predicts European Index options and S\&P 500 European call options more accurately than standard NN (Gencay \& Gibson, 2007).

\section{Hybrid NN -}

NN with Black Scholes - The research study by Blynski, and Faseruk (2006) compared the effectiveness of option price forecasting using the traditional Black Scholes model with NN (hybrid NN) and standard NN. Likewise, the research study by Chen and Sutcliffe (2012) confirms that a hybrid NN along with Black Scholes predicts accurately than the standard NN or Black Scholes model individually. Similarly, Sperckelsen et al. (2014) used Black Scholes model variables in predicting option prices of currency futures (EUR/USD and concluded that the hybrid model is better than the theoretical option pricing model and MLP.

NN with Black Scholes \& Wavelet - Zapart (2003) in his research study uses Wavelet along with NN and Black Scholes. Wavelet is a mathematical advancement that addresses oscillations that decays quickly in a data set. The study found that Black Scholes NN with Wavelet predicts superior when analyzing option prices.

Hybrid Black Scholes NN with stochastic volatility - Stochastic volatility represents the nature of volatility fluctuating over time. The research study by Gencay and Gibson (2007) found that the hybrid NN model with stochastic volatility predicts better than the standard NN Model while predicting European stock index options.

\section{b) Futures -}

Standard NN - Model-free NN, without the attributes of financial theories, also performs significantly in the case of predicting currency futures prices. A model-free NN is used to predict the high-frequency currency futures and the predictability power is better than the closed-form financial model (Sperckelsen et al., 2014).

\section{Optimized NN -}

Multilayer perceptron (MLP) - MLP commonly represents a feed-forward NN with three layers. MLP is used to predict the commodity futures to hedge against corn and ethanol spreads and found to be accurate in prediction (Dunis et al., 2015). Likewise, Karathanasapoulos et al. (2016) used MLP in gasoline futures contracts.

Higher-order NN (HONN) -HONN utilizes a higher combination of NN inputs. The research study of Dunis et al. (2015) compared the performance of HONN with MLP. The study concludes that MLP and HONN are superior in predicting with leveraging option. Sermpinis et al. (2013) tested HONN in predicting index futures. Likewise, Karathanasapoulos et al. (2016), in their research study used HONN to predict gasoline futures contracts.

Radical base function neural network (RBFNN)- Karathanasapoulos et al. (2016) used a radical basic function neural network (RBFNN) to improve the trading performance of futures. RBFNN transforms the input signal into another form, which can be then feed into the network to get linear separability. The study concludes that RBF NN is superior in both trading performance and statistical accuracy. 


\section{Hybrid NN -}

Feature transformed NN - Kim (2004) used feature transformed NN, including domain-specific factors like relative strength index to predict the futures prices and found that feature transformed $\mathrm{NN}$ predicts accurately than the linear models and concludes that incorporating domain knowledge in NN architecture improves performance.

\section{Shares \& Indices -}

Standard NN -

Haefke and Helmenstien (2002) used NN in forecasting indices and inferred that applicability of information criteria is important in the selection of NN. In contrast, Moreno and Olmeda (2007) claim that $\mathrm{NN}$ is not superior in predicting the stock markets to the linear models.

\section{Optimized NN -}

NN with Statistical optimization - LV D et al. (2020) used principal component analysis (PCA), Least absolute shrinkage and selection operators (LASSO), classification and regression trees (CART), and Piecewise linear representation methods (PLR) to optimize NN. As a result, there no significant improvement in NN incorporating the features, however, NN with PLR, resulted in an improvement in profit through better forecast ability.

Back propagation (BPNN) and Piecewise linear representation (PLR) - In a research study by Chang et al. (2009), it is found that BPNN along with PLR consistently created good results for predicting upward, steady, and downward trends of stock prices.

\section{Hybrid NN -}

NN with Fama French five-factor model - Besides customizing the NN, researchers have used financial models like Fama and French five-factor model with NN and found improvement in the profitability of investors in both linear and nonlinear data (Jan \& Ayub, 2019).

NN with GARCH model-Ozbey and Paksoy (2020) combined GARCH with NN and compared the performance of the hybrid model with the classic GARCH model. The study found that the hybrid model is superior in predicting volatility to the classic GARCH.

NN with Top-down theory, technical analysis, and dynamic time series methods -Huang, G.,Huang,GB., Shiji, and Youa (2014) used integrated models using conventional top-down trading theory, technical analysis, and dynamic time series methods and concludes that the hybrid system gives remarkable investment returns and demonstrates promising potential tools for stock market forecasting.

\section{Other related financial forecasts - Optimized NN -}

Back propagation NN (BPNN) - Chiang et al. (1996) used BPNN to predict the Net Asset Value (NAV) of mutual funds and found that BPNN outperforms the regression model. Jain and Nag (1995) predicted the prices of initial public offering (IPO) using BPNN and found significant economic benefits in BPNN. 
Multi-layer perception (MLP) - Indroa et al. (1999) used MLP and compared it with stepwise linear regression. The results show that MLP is superior to the linear model.

General regression NN (GRNN) -Barnes and Lee (2009) used GRNN in analyzing the effect of macroeconomic and firm-specific factors in determining shareholder wealth.

Multivariate NN -Wie et al. (2004) used Multivariate NN to predict earning per share in comparison with univariate and multivariate linear models incorporating fundamental accounting variables and found that $\mathrm{NN}$ along with accounting variables predicts more accurately than linear forecasting models.

Table 3. NN research in Investment prediction

\begin{tabular}{|c|c|c|c|c|c|c|c|c|c|}
\hline & Author(s) & Purpose & NN Model & Sample & Period & Predictors & Comparison & $\begin{array}{l}\text { R Squared } \\
\text { Value }\end{array}$ & Accuracy \\
\hline 1 & $\begin{array}{l}\text { Kaastra and } \\
\text { Boyd (1995) }\end{array}$ & $\begin{array}{l}\text { Forecastin } \\
\mathrm{g} \\
\text { Economic } \\
\text { time- } \\
\text { series data }\end{array}$ & $\begin{array}{l}\text { Back } \\
\text { Propagation } \\
\text { NN }\end{array}$ & $\begin{array}{l}\text { Conceptu } \\
\text { al Paper }\end{array}$ & - & 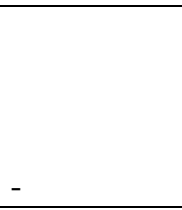 & 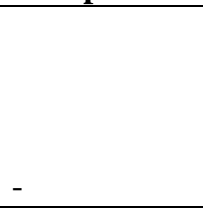 & 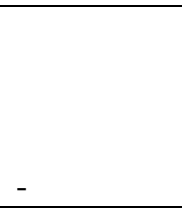 & - \\
\hline 2 & $\begin{array}{l}\text { Lin and Yeh } \\
(2009)\end{array}$ & $\begin{array}{l}\text { Forecastin } \\
\text { g Option } \\
\text { Prices }\end{array}$ & $\begin{array}{l}\text { Back } \\
\text { Propagation } \\
\text { NN }\end{array}$ & $\begin{array}{l}15582 \\
\text { call } \\
\text { option } \\
\text { price data } \\
\text { points }\end{array}$ & $\begin{array}{l}2003- \\
2004\end{array}$ & $\begin{array}{l}\text { Black } \\
\text { Scholes } \\
\text { variables }\end{array}$ & & - & $\begin{array}{l}\text { MAPE } \\
5.2534\end{array}$ \\
\hline 3 & $\begin{array}{l}\text { Hutchison et } \\
\text { al. (1994) }\end{array}$ & $\begin{array}{l}\text { Pricing } \\
\text { and } \\
\text { Hedging } \\
\text { derivative } \\
\text { securities }\end{array}$ & $\begin{array}{l}\text { Ordinary } \\
\text { Least squares, } \\
\text { Radical Basis } \\
\text { functions } \\
\text { network, } \\
\text { Multi-layer } \\
\text { Perceptron, } \\
\text { Projection } \\
\text { Pursuit }\end{array}$ & $\begin{array}{l}\text { S\&P } 500 \\
\text { Future } \\
\text { and } \\
\text { options }\end{array}$ & $\begin{array}{l}1987- \\
1991\end{array}$ & $\begin{array}{l}\text { Black } \\
\text { Scholes } \\
\text { variables }\end{array}$ & $\begin{array}{l}\text { No } \\
\text { significant } \\
\text { difference } \\
\text { between } \\
\text { models }\end{array}$ & 84.76 & - \\
\hline 4 & $\begin{array}{l}\text { Kohler et al. } \\
(2010)\end{array}$ & $\begin{array}{l}\text { Pricing of } \\
\text { American } \\
\text { Options }\end{array}$ & $\begin{array}{l}\text { Least square } \\
\text { NN }\end{array}$ & $\begin{array}{l}\text { Monte } \\
\text { Carlo } \\
\text { Simulated } \\
\text { Data }\end{array}$ & - & - & - & - & - \\
\hline 5 & $\begin{array}{l}\text { Gradojevic } \\
\text { et al. }(2009)\end{array}$ & $\begin{array}{l}\text { Pricing } \\
\text { European } \\
\text { Call } \\
\text { options }\end{array}$ & $\begin{array}{l}\text { Modular NN } \\
\text { \& Black } \\
\text { Scholes NN }\end{array}$ & $\begin{array}{l}\text { S\&P-500 } \\
\text { index } \\
\text { European } \\
\text { call } \\
\text { option } \\
\text { prices, } \\
\text { Chicago } \\
\text { Board } \\
\text { Options } \\
\text { Exchange }\end{array}$ & $\begin{array}{l}1987 \text { - } \\
1994\end{array}$ & $\begin{array}{l}\text { Black } \\
\text { Scholes } \\
\text { variables }\end{array}$ & $\begin{array}{l}\text { BS NN } \\
\text { Model > } \\
\text { Modular NN }\end{array}$ & - & $\begin{array}{l}\text { MAPE } \\
1.87 \\
\end{array}$ \\
\hline 6 & $\begin{array}{l}\text { Gencay and } \\
\text { Gibson } \\
(2007)\end{array}$ & $\begin{array}{l}\text { Pricing } \\
\text { European } \\
\text { Stock } \\
\text { Index } \\
\text { options } \\
\end{array}$ & $\begin{array}{l}\text { Feedforward } \\
\text { NN }\end{array}$ & $\begin{array}{l}\text { S\&P 500 } \\
\text { index9 } \\
\text { options } \\
\text { from the } \\
\text { Berkeley }\end{array}$ & $\begin{array}{l}1989- \\
1991\end{array}$ & $\begin{array}{l}\text { Price of the } \\
\text { underlying, } \\
\text { strike price, } \\
\text { volatility, } \\
\text { interest }\end{array}$ & $\begin{array}{l}\text { FFNN > } \\
\text { Stochastic } \\
\text { volatility } \\
\text { (SV) and } \\
\text { stochastic }\end{array}$ & - & - \\
\hline
\end{tabular}




\begin{tabular}{|c|c|c|c|c|c|c|c|c|c|}
\hline & & & & $\begin{array}{l}\text { Option } \\
\text { Database }\end{array}$ & & $\begin{array}{l}\text { rate, time } \\
\text { to maturity }\end{array}$ & $\begin{array}{l}\text { volatility } \\
\text { random jump } \\
\text { (SVJ), }\end{array}$ & & \\
\hline 7 & $\begin{array}{l}\text { Blensky and } \\
\text { Fasurek } \\
(2006)\end{array}$ & $\begin{array}{l}\text { Comparin } \\
\text { g Option } \\
\text { prices } \\
\text { forecast of } \\
\text { NN with } \\
\text { Black } \\
\text { Scholes } \\
\text { model } \\
\end{array}$ & $\begin{array}{l}\text { Back } \\
\text { Propagation } \\
\text { NN }\end{array}$ & $\begin{array}{l}64,280 \\
\text { OEX } 100 \\
\text { Index call } \\
\text { option }\end{array}$ & $\begin{array}{l}1986- \\
1993 \\
\end{array}$ & - & $\begin{array}{l}\mathrm{NN}>\text { Black } \\
\text { Scholes } \\
\text { model }\end{array}$ & - & - \\
\hline 8 & $\begin{array}{l}\text { Chen and } \\
\text { Sutcliffe } \\
\text { (2012) }\end{array}$ & $\begin{array}{l}\text { Pricing } \\
\text { and } \\
\text { hedging } \\
\text { short } \\
\text { sterling } \\
\text { options } \\
\end{array}$ & $\begin{array}{l}\text { Standard NN, } \\
\text { Modified } \\
\text { Black Model } \\
\text { NN, and } \\
\text { Hybrid NN }\end{array}$ & $\begin{array}{l}\text { Short } \\
\text { sterling } \\
\text { futures } \\
\text { traded on } \\
\text { NYSE }\end{array}$ & $\begin{array}{l}2012 \\
\text { (Quarterl } \\
\text { y expiry } \\
\text { cycle) }\end{array}$ & $\begin{array}{l}\text { Ask, bid, } \\
\text { trade, } \\
\text { spread } \\
\text { trade, and } \\
\text { block trade, }\end{array}$ & $\begin{array}{l}\text { Hybrid NN > } \\
\text { Modified } \\
\text { Black model, } \\
\text { Standard NN }\end{array}$ & $r=98.6864$ & - \\
\hline 9 & $\begin{array}{l}\text { Sperckelsen } \\
\text { et al. (2014) }\end{array}$ & $\begin{array}{l}\text { Realtime } \\
\text { pricing of } \\
\text { options on } \\
\text { currency } \\
\text { futures }\end{array}$ & $\begin{array}{l}\text { Model-free } \\
\text { option pricing } \\
\text { NN, Multi- } \\
\text { Layer } \\
\text { Perceptron }\end{array}$ & $\begin{array}{l}\text { EUR/US } \\
\text { D option } \\
\text { on } \\
\text { currency } \\
\text { future } \\
\text { from } \\
\text { Chicago } \\
\text { Mercantil } \\
\text { e } \\
\text { Exchange } \\
\text { (CME) }\end{array}$ & 2012 & $\begin{array}{l}\text { Futures } \\
\text { price, } \\
\text { Strike } \\
\text { price, } \\
\text { Expiration } \\
\text { time, Risk- } \\
\text { free rate, } \\
\text { Asset } \\
\text { volatility }\end{array}$ & $\begin{array}{l}\text { Hybrid NN > } \\
\text { theoretical } \\
\text { option pricing } \\
\text { model }\end{array}$ & $99 \%$ & $\begin{array}{l}\text { MAPE } \\
0.3146\end{array}$ \\
\hline $\begin{array}{l}1 \\
0 \\
\end{array}$ & $\begin{array}{l}\text { Zapart } \\
(2003) \\
\end{array}$ & $\begin{array}{l}\text { Pricing } \\
\text { European } \\
\text { and } \\
\text { American } \\
\text { Call } \\
\text { options } \\
\end{array}$ & $\begin{array}{l}\text { NN with } \\
\text { Binomial } \\
\text { trees and } \\
\text { Wavelets, NN } \\
\text { with Genetic } \\
\text { algorithm and } \\
\text { Black Scholes } \\
\text { model }\end{array}$ & $\begin{array}{l}\text { Options } \\
\text { prices as } \\
\text { quoted on } \\
\text { the } \\
\text { Chicago } \\
\text { Board } \\
\text { Options } \\
\text { Exchange } \\
\text { are used } \\
\end{array}$ & 2003 & $\begin{array}{l}\text { Time to } \\
\text { expiry, } \\
\text { current } \\
\text { stock price, } \\
\text { risk-free } \\
\text { rate }\end{array}$ & 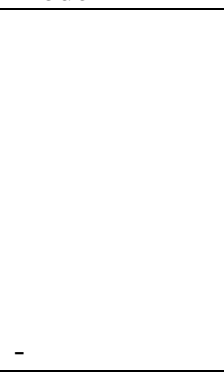 & - & $x_{1}$ \\
\hline $\begin{array}{l}1 \\
1\end{array}$ & $\begin{array}{l}\text { Dunis et al. } \\
\text { (2015) }\end{array}$ & $\begin{array}{l}\text { Modeling } \\
\text { corn/ethan } \\
\text { ol crush } \\
\text { spread }\end{array}$ & $\begin{array}{l}\text { MLP, HONN, } \\
\text { GPA }\end{array}$ & $\begin{array}{l}\text { Ethanol } \\
\text { futures } \\
\text { contract } \\
\text { traded in } \\
\text { Chicago } \\
\text { Board }\end{array}$ & $\begin{array}{l}2005- \\
2010\end{array}$ & Leverage & $\begin{array}{l}\text { GPA > } \\
\text { HONN, MLP }\end{array}$ & - & - \\
\hline $\begin{array}{l}1 \\
3 \\
\end{array}$ & $\begin{array}{l}\text { Sermpinis et } \\
\text { al. (2013) }\end{array}$ & $\begin{array}{l}\text { The } \\
\text { forecastin } \\
\text { g FTSE } \\
100 \\
\text { futures }\end{array}$ & $\begin{array}{l}\text { Higher-order } \\
\text { NN, Multi- } \\
\text { Layer } \\
\text { Perceptron, } \\
\text { Recurrent } \\
\text { neural } \\
\text { networks }\end{array}$ & $\begin{array}{l}\text { FTSE } 100 \\
\text { futures }\end{array}$ & $\begin{array}{l}2007- \\
2008\end{array}$ & $\begin{array}{l}\text { Realized } \\
\text { daily } \\
\text { returns (21 } \\
\text { days) }\end{array}$ & $\begin{array}{l}\text { HONN> } \\
\text { MLP, RNN }\end{array}$ & - & $18.85 \%$ \\
\hline $\begin{array}{l}1 \\
4 \\
\end{array}$ & $\begin{array}{l}\text { Karathanaso } \\
\text { poulos et al. } \\
\text { (2016) }\end{array}$ & $\begin{array}{l}\text { Modeling } \\
\text { crack } \\
\text { spread }\end{array}$ & $\begin{array}{l}\text { RBF, PSO, } \\
\text { MLP }\end{array}$ & - & $\begin{array}{l}2005- \\
2015\end{array}$ & $\begin{array}{l}20 \text { ARIMA } \\
\text { and } 10 \\
\text { GARCH } \\
\text { models }\end{array}$ & $\begin{array}{l}\text { PSORBF > } \\
\text { MLP }\end{array}$ & - & - \\
\hline 1 & Kim (2004) & Future & Feature & Korean & May to & Positive & Feature & - & - \\
\hline
\end{tabular}




\begin{tabular}{|c|c|c|c|c|c|c|c|c|c|}
\hline 5 & & $\begin{array}{l}\text { price } \\
\text { prediction }\end{array}$ & $\begin{array}{l}\text { transformed } \\
\text { ANN based } \\
\text { on domain } \\
\text { knowledge }\end{array}$ & $\begin{array}{l}\text { stock } \\
\text { index } \\
\text { (KOSPI) }\end{array}$ & $\begin{array}{l}\text { Novemb } \\
\text { er } 1996\end{array}$ & $\begin{array}{l}\text { volume } \\
\text { index, Rate } \\
\text { of Change, } \\
\text { Momentum } \\
\text {, etc. }\end{array}$ & $\begin{array}{l}\text { transformed } \\
\text { ANN > } \\
\text { Linear ANN }\end{array}$ & & \\
\hline $\begin{array}{l}1 \\
6\end{array}$ & $\begin{array}{l}\text { Haefke and } \\
\text { Helmenstien } \\
(2002)\end{array}$ & $\begin{array}{l}\text { Index } \\
\text { Forecastin } \\
\mathrm{g} \text { and } \\
\text { Model } \\
\text { Selection }\end{array}$ & $\begin{array}{l}\text { Feedforward } \\
\text { NN }\end{array}$ & $\begin{array}{l}\text { Austrian } \\
\text { Traded } \\
\text { Index } \\
\text { (ATX) }\end{array}$ & $\begin{array}{l}2 \\
\text { Novemb } \\
\text { er } \\
1992 \text { to } \\
14 \\
\text { October } \\
1994\end{array}$ & $\begin{array}{l}\text { Geometric } \\
\text { mean, } \\
\text { arithmetic } \\
\text { mean }\end{array}$ & $\begin{array}{l}\text { The proposed } \\
\text { integrated } \\
\text { model shows } \\
\text { significant } \\
\text { performanc }\end{array}$ & 0.041 & $\begin{array}{l}\text { AMAPE = } \\
1.862\end{array}$ \\
\hline $\begin{array}{l}1 \\
7\end{array}$ & $\begin{array}{l}\text { Moreno and } \\
\text { Olmeda } \\
(2007)\end{array}$ & $\begin{array}{l}\text { Predictabi } \\
\text { lity of } \\
\text { emerging } \\
\text { and } \\
\text { developed } \\
\text { stock } \\
\text { markets } \\
\text { using NN }\end{array}$ & Standard NN & $\begin{array}{l}\text { 49 MSCI } \\
\text { (Morgan } \\
\text { Stanley } \\
\text { Capital } \\
\text { Internatio } \\
\text { nal) } \\
\text { indexes }\end{array}$ & $\begin{array}{l}\text { March } \\
1995 \text { to } \\
\text { March of } \\
2001 \\
(1560 \\
\text { daily } \\
\text { observati } \\
\text { ons) }\end{array}$ & $\begin{array}{l}\text { index } \\
\text { returns, } \\
\text { daily and } \\
\text { weekly }\end{array}$ & $\begin{array}{l}\mathrm{NN} \text { is not } \\
\text { superior to } \\
\text { the linear } \\
\text { models }\end{array}$ & - & 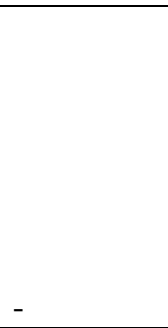 \\
\hline $\begin{array}{l}1 \\
8\end{array}$ & $\begin{array}{l}\text { LV D et al. } \\
(2020)\end{array}$ & $\begin{array}{l}\text { Dimensio } \\
\text { nality } \\
\text { reduction } \\
\text { in stock } \\
\text { trading }\end{array}$ & $\begin{array}{l}\text { MLP, Deep } \\
\text { Belief } \\
\text { Network } \\
\text { (DBN), } \\
\text { Stacked } \\
\text { Auto- } \\
\text { Encoders } \\
\text { (SAE), RNN, } \\
\text { LSTM, Gated } \\
\text { Recurrent } \\
\text { Unit (GRU) } \\
\end{array}$ & $\begin{array}{l}\text { US } \\
\text { SPICS } \\
\text { and the } \\
\text { Chinese } \\
\text { CSICS }\end{array}$ & $\begin{array}{l}\text { Past } \\
2000 \\
\text { trading } \\
\text { days of } \\
\text { SPICS } \\
\text { and } \\
\text { CSICS } \\
\text { before } \\
\text { Decembe } \\
\text { r } \\
31,2017 \\
\end{array}$ & $\begin{array}{l}44 \\
\text { technical, } \\
\text { Volatility, } \\
\text { Psychologi } \\
\text { cal, cash } \\
\text { flow } \\
\text { indicators. }\end{array}$ & LASSO NN & - & - \\
\hline $\begin{array}{l}1 \\
9\end{array}$ & $\begin{array}{l}\text { Chang et al. } \\
\text { (2009) }\end{array}$ & $\begin{array}{l}\text { Stock } \\
\text { Trading } \\
\text { Points } \\
\text { Prediction }\end{array}$ & $\begin{array}{l}\text { BPNN, GA, } \\
\text { PLR }\end{array}$ & $\begin{array}{l}\text { Stock } \\
\text { Prices }\end{array}$ & $\begin{array}{l}2004 / 01 / \\
02 \text { to } \\
2006 / 04 / \\
12\end{array}$ & $\begin{array}{l}\text { Moving } \\
\text { average, } \\
\text { Bias, RSI, } \\
\text { ninety days } \\
\text { stochastic } \\
\text { line, etc. }\end{array}$ & $\begin{array}{l}\text { PLR +GA } \\
\text { improves } \\
\text { Profitability }\end{array}$ & - & - \\
\hline $\begin{array}{l}2 \\
0 \\
\end{array}$ & $\begin{array}{l}\text { Jan and } \\
\text { Ayub (2019) }\end{array}$ & $\begin{array}{l}\text { Improving } \\
\text { the } \\
\text { predictabil } \\
\text { ity of } \\
\text { Fama } \\
\text { French } \\
\text { five-factor } \\
\text { model }\end{array}$ & Standard NN & $\begin{array}{l}\text { Manufact } \\
\text { uring } \\
\text { companie } \\
\text { s in } \\
\text { Pakistan } \\
\text { Stock } \\
\text { Exchange }\end{array}$ & $\begin{array}{l}2000 \text { to } \\
2015\end{array}$ & $\begin{array}{l}\text { Market } \\
\text { cap, } \\
\text { BV/MV } \\
\text { ratio, \% in } \\
\text { total assets, } \\
\text { and EBIT }\end{array}$ & $\begin{array}{l}\text { NN improves } \\
\text { FF model }\end{array}$ & $r=0.99989$ & $\begin{array}{l}\text { MSE = } \\
0.0012\end{array}$ \\
\hline $\begin{array}{l}2 \\
1 \\
\end{array}$ & $\begin{array}{l}\text { Ozbey and } \\
\text { Paksoy } \\
(2020)\end{array}$ & $\begin{array}{l}\text { Estimatio } \\
\mathrm{n} \text { of index } \\
\text { returns } \\
\text { with } \\
\text { GARCH } \\
\text { and NN }\end{array}$ & $\begin{array}{l}\text { Hybrid NN, } \\
\text { Exp GARCH, } \\
\text { and Nor. } \\
\text { Distrn }\end{array}$ & $\begin{array}{l}\text { Borsa } \\
\text { Istanbul } \\
100 \text { price } \\
\text { Index }\end{array}$ & $\begin{array}{l}2017- \\
2018\end{array}$ & $\begin{array}{l}\text { Borsa } \\
\text { Istanbul } \\
100 \text { Index } \\
\text { value }\end{array}$ & $\begin{array}{l}\text { Hybrid NN + } \\
\text { GARCH > } \\
\text { Hybrid NN + } \\
\text { normal } \\
\text { distribution }\end{array}$ & - & $\begin{array}{l}\text { MSE = } \\
0.015926\end{array}$ \\
\hline $\begin{array}{l}2 \\
2\end{array}$ & $\begin{array}{l}\text { Huang, } \\
\text { Huang, } \\
\text { Shiji, and } \\
\text { Youa (2014) }\end{array}$ & $\begin{array}{l}\text { Integrated } \\
\text { data } \\
\text { mining in } \\
\text { stock }\end{array}$ & $\begin{array}{l}\text { Top-down } \\
\text { trading theory } \\
+ \text { ANN + } \\
\text { technical }\end{array}$ & $\begin{array}{l}\text { Taiwan } \\
\text { Semicond } \\
\text { uctor } \\
\text { Manufact }\end{array}$ & $\begin{array}{l}2011- \\
2013\end{array}$ & $\begin{array}{l}\text { Stochastic } \\
\text { KD, } \\
\text { William } \\
\% \text { R, RSI, }\end{array}$ & $\begin{array}{l}\text { Integrated } \\
\text { NN model } \\
\text { improves } \\
\text { stock }\end{array}$ & - & $\begin{array}{l}\text { True } \\
\text { positive = } \\
98.50 \%\end{array}$ \\
\hline
\end{tabular}




\begin{tabular}{|c|c|c|c|c|c|c|c|c|c|}
\hline & & $\begin{array}{l}\text { forecastin } \\
\mathrm{g}\end{array}$ & $\begin{array}{l}\text { analysis + } \\
\text { dynamic time } \\
\text { series + and } \\
\text { Bayesian } \\
\text { probability }\end{array}$ & $\begin{array}{l}\text { uring } \\
\text { Company } \\
\text { and } \\
\text { Evergreen } \\
\text { Marine } \\
\text { Corporati } \\
\text { on } \\
\end{array}$ & & $\begin{array}{l}\text { PSY line, } \\
\text { ADX, MA, } \\
\text { MACD }\end{array}$ & forecasting & & \\
\hline $\begin{array}{l}2 \\
3 \\
\end{array}$ & $\begin{array}{l}\text { Chiang et al. } \\
(1996)\end{array}$ & $\begin{array}{l}\text { Mutual } \\
\text { Fund } \\
\text { NAV } \\
\text { forecastin } \\
\mathrm{g}\end{array}$ & BPNN & $\begin{array}{l}6 \text { Year } \\
\text { economic } \\
\text { variables } \\
\text { and } 101 \\
\text { US } \\
\text { mutual } \\
\text { funds }\end{array}$ & $\begin{array}{l}1981- \\
1986\end{array}$ & $\begin{array}{l}\text { GNP, } \\
\text { Consumpti } \\
\text { on, } \\
\text { Investment, } \\
\text { CPI, } \\
\text { Money } \\
\text { supply, } \\
\text { unemploy } \\
\text { ment, T- } \\
\text { bill, Long } \\
\text { term rate } \\
\end{array}$ & $\begin{array}{l}\text { BPNN > } \\
\text { Linear \& } \\
\text { Non-Linear } \\
\text { regression }\end{array}$ & 0.989 & $\begin{array}{l}\text { MAPE = } \\
8.76\end{array}$ \\
\hline $\begin{array}{l}2 \\
4\end{array}$ & $\begin{array}{l}\text { Jain and Nag } \\
\text { (1995) }\end{array}$ & $\begin{array}{l}\text { Predicting } \\
\text { IPO } \\
\text { pricing }\end{array}$ & FFNN & $\begin{array}{l}552 \text { IPOs } \\
\text { in the } \\
\text { United } \\
\text { States }\end{array}$ & $\begin{array}{l}1980- \\
1990\end{array}$ & $\begin{array}{l}11 \\
\text { variables } \\
\text { [Size, } \\
\text { Underwrite } \\
\text { r, sales, } \\
\text { ROA, ROI, } \\
\text { Assets } \\
\text { turnover, } \\
\text { etc.] }\end{array}$ & - & - & - \\
\hline $\begin{array}{l}2 \\
5\end{array}$ & $\begin{array}{l}\text { Indroa et al. } \\
\text { (1999) }\end{array}$ & $\begin{array}{l}\text { Predicting } \\
\text { mutual } \\
\text { fund } \\
\text { performan } \\
\text { ce }\end{array}$ & MLP & $\begin{array}{l}\text { Morningst } \\
\text { ar Mutual } \\
\text { Funds } \\
\text { On-Disc } \\
\text { database }\end{array}$ & $\begin{array}{l}1993- \\
1995\end{array}$ & $\begin{array}{l}\text { Annualized } \\
\text { return, } \\
\text { turnover, } \\
\mathrm{P} / \mathrm{E}, \mathrm{P} / \mathrm{B} \text {, } \\
\text { Mar.Cap }\end{array}$ & $\begin{array}{l}\text { MLP > } \\
\text { Linear } \\
\text { models }\end{array}$ & - & $\begin{array}{l}\text { MAPE = } \\
4.88\end{array}$ \\
\hline $\begin{array}{l}2 \\
6 \\
\end{array}$ & $\begin{array}{l}\text { Barnes and } \\
\text { Lee }(2009)\end{array}$ & $\begin{array}{l}\text { Effects of } \\
\text { Macroeco } \\
\text { nomic- } \\
\text { Firm- } \\
\text { Specific } \\
\text { Factors } \\
\text { on } \\
\text { Sharehold } \\
\text { er Wealth }\end{array}$ & $\begin{array}{l}\text { General } \\
\text { regression } \\
\text { NN (GRNN) }\end{array}$ & $\begin{array}{l}\text { Miscellan } \\
\text { eous } \\
\text { Industrials } \\
\text { in } \\
\text { the } \\
\text { Australian } \\
\text { Stock } \\
\text { Market }\end{array}$ & 2007 & $\begin{array}{l}\text { D/E, Gross } \\
\text { margin, } \\
\text { Debt to } \\
\text { cash, EVA, } \\
\text { EPS, } \\
\text { WACC } \\
\text { funds, } \\
\text { ROIC } \\
\end{array}$ & $\begin{array}{l}\text { ANN is } \\
\text { effective in } \\
\text { the prediction }\end{array}$ & 0.0548 & $\begin{array}{l}\text { MAE = } \\
37.649\end{array}$ \\
\hline $\begin{array}{l}2 \\
7 \\
\end{array}$ & $\begin{array}{l}\text { Wie et al. } \\
(2004)\end{array}$ & $\begin{array}{l}\text { NN model } \\
\text { for EPS } \\
\text { forecastin } \\
\mathrm{g}\end{array}$ & $\begin{array}{l}\text { Univariate- } \\
\text { NN and } \\
\text { multivariate } \\
\text { NN }\end{array}$ & $\begin{array}{l}\text { Quarterly } \\
\text { EPS of } \\
283 \\
\text { companie } \\
\text { s in SEC }\end{array}$ & $\begin{array}{l}1992- \\
2002\end{array}$ & $\begin{array}{l}\text { Inventory, } \\
\text { A/R, } \\
\text { capital } \\
\text { expenditure } \\
\text {, gross } \\
\text { margin, } \\
\text { Sel.Adm } \\
\text { exp, Tax } \\
\text { rate, labour } \\
\text { force }\end{array}$ & $\begin{array}{l}\text { NN models > } \\
\text { Linear } \\
\text { models }\end{array}$ & - & $\begin{array}{l}\text { MAPE = } \\
0.362\end{array}$ \\
\hline
\end{tabular}




\section{Credit Risk Prediction:}

Credit evaluation -

Optimized NN -

Bayesian regularized NN (BRNN) - Sariev and Germano (2020) used BRNN to Predict the Probability of Default and found BRNN superior in prediction.

Back propagation NN (BPNN) - Loss-given default (LGD) is used in credit risk assessment. LGD is the share of an asset that is lost if a borrower default. Loterman et al. (2012) compared the nonlinear techniques with the linear counterparts in predicting LGD of major international banks.

Feed forward NN (FFNN) - Qi and Zhao (2011) found that nonparametric method like FFNN and regression tree predicts the LGD accurately both in and out of sample than their parametric counterparts. Cifter et al. (2009) investigated the relationship between industrial production and credit defaults (nonperforming loans) using FFNN based on wavelet decomposition.

Fuzzy mathematical model -Aiqun et al. (2020) applied NN in risk assessment of logistic finance using back propagation NN and Fuzzy mathematical model. The study found that NN with the fuzzy mathematical model is accurate in risk assessment. Further, Baesens et al. (2003) provided a table with a graphical format that facilitates easy consultation to interpret the NN results.

\section{Credit Scoring -}

\section{Standard NN -}

Chikolwa and Chan (2008) compared NN with ordinal regression (OR) to study the determinants of Commercial Mortgage-Backed Securities (CMBS) and concluded that NN is superior in prediction to OR. Trinkle and Baldwin (2007) applied NN in credit evaluation for loan finance and concluded that NN can be used in the credit scoring process with caution because of its hidden nature.

\section{Optimized NN -}

Backpropagation NN- Hajek (2011) applied NN to rate the United States municipalities in the state of Connecticut and found a higher accuracy of NN in classifying the municipalities with a limited subset of variables. Zan et al. (2004) compared the performance of Support vector machines (SVM) with back propagation NN (BPNN) on the credit rating of companies and found that both SVM and BPNN have the same accuracy in predicting the credit rating.

Table 4. NN literatures in Credit Risk analysis

\begin{tabular}{|c|c|c|c|c|c|c|c|c|c|}
\hline & Author(s) & Purpose & NN Model & Sample & $\begin{array}{l}\text { Output } \\
\text { Variable }\end{array}$ & Predictors & Comparison & $\begin{array}{l}\mathbf{R} \\
\text { Squared } \\
\text { Value } \\
\end{array}$ & Accuracy \\
\hline 1 & $\begin{array}{l}\text { Sariev and } \\
\text { Germano } \\
(2020)\end{array}$ & $\begin{array}{l}\text { Estimatio } \\
\mathrm{n} \text { of the } \\
\text { probabilit } \\
\text { y of } \\
\text { default }\end{array}$ & $\begin{array}{l}\text { BRNN, } \\
\text { BPNN }\end{array}$ & $\begin{array}{l}\text { East } \\
\text { Europea } \\
\text { n, } \\
\text { German, } \\
\text { and } \\
\text { Polish }\end{array}$ & $\begin{array}{l}2007- \\
2012 \\
(\mathrm{EE}) \\
2007- \\
2013(\mathrm{P})\end{array}$ & $\begin{array}{l}\text { Payables } \\
\text { turnover, } \\
\text { ROA, cash } \\
\text { ratio, } \\
\text { sales/total } \\
\text { assets, }\end{array}$ & $\begin{array}{l}\text { BRNN > } \\
\text { BPNN }\end{array}$ & - & - \\
\hline
\end{tabular}




\begin{tabular}{|c|c|c|c|c|c|c|c|c|c|}
\hline & & & & data & & $\begin{array}{l}\mathrm{LA} / \mathrm{TA}, \\
\text { interest } \\
\text { coverage }\end{array}$ & & & \\
\hline 2 & $\begin{array}{l}\text { Lotterman } \\
\text { et al. } \\
\text { (2012)\# }\end{array}$ & $\begin{array}{l}\text { Benchmar } \\
\text { king } \\
\text { regression } \\
\text { algorithms } \\
\text { for loss } \\
\text { given } \\
\text { default } \\
\text { modeling }\end{array}$ & $\begin{array}{l}\text { NN, SVM, } \\
\text { and OLS. }\end{array}$ & $\begin{array}{l}\text { six LGD } \\
\text { datasets } \\
\text { from } \\
\text { internati } \\
\text { onal } \\
\text { banks }\end{array}$ & - & - & $\begin{array}{l}\text { SVM, NN > } \\
\text { Linear Models }\end{array}$ & 0.1295 & $\begin{array}{l}\mathrm{MAE}= \\
0.3118\end{array}$ \\
\hline 3 & $\begin{array}{l}\text { Qi and } \\
\text { Zhao } \\
(2011) \#\end{array}$ & $\begin{array}{l}\text { Comparis } \\
\text { on of } \\
\text { modeling } \\
\text { methods } \\
\text { for Loss } \\
\text { Given } \\
\text { Default } \\
\end{array}$ & $\begin{array}{l}\text { OLS, } \\
\text { fractional } \\
\text { response } \\
\text { regression } \\
\text { (FRR), } \\
\text { inverse } \\
\text { Gaussian } \\
\text { regression } \\
\text { (IGR), and } \\
\text { inverse } \\
\text { Gaussian } \\
\text { regression } \\
\text { with beta } \\
\text { transforma } \\
\text { tion (IGR- } \\
\text { BT), and } \\
\text { regression } \\
\text { tree (RT), } \\
\text { NN }\end{array}$ & $\begin{array}{l}3751 \\
\text { defaulte } \\
\text { d } \\
\text { securitie } \\
\text { s in the } \\
\text { US, } \\
\text { Moody' } \\
\text { s } \\
\text { Ultimate } \\
\text { Recover } \\
\text { y } \\
\text { Databas } \\
\text { e } \\
\end{array}$ & $\begin{array}{l}1985 \text { to } \\
2008\end{array}$ & - & $\begin{array}{l}\mathrm{NN}, \\
\text { regression tree } \\
>\text { linear } \\
\text { regression, } \\
\text { fractional } \\
\text { response, OLS }\end{array}$ & 0.576 & - \\
\hline 4 & $\begin{array}{l}\text { Cifter et } \\
\text { al. }(2009)\end{array}$ & $\begin{array}{l}\text { Examine } \\
\text { the } \\
\text { relationshi } \\
\text { p between } \\
\text { industrial } \\
\text { productio } \\
\mathrm{n} \text { and } \\
\text { credit } \\
\text { defaults }\end{array}$ & $\begin{array}{l}\text { FFNN } \\
\text { using } \\
\text { Wavelet } \\
\text { decomposi } \\
\text { tion }\end{array}$ & $\begin{array}{l}83 \\
\text { monthly } \\
\text { observat } \\
\text { ions } \\
\text { Industria } \\
1 \\
\text { producti } \\
\text { on and } \\
\text { credit } \\
\text { default } \\
\text { rates are } \\
\text { from } \\
\text { Central } \\
\text { Bank of } \\
\text { Turkey }\end{array}$ & $\begin{array}{l}2001 \text { to } \\
2007\end{array}$ & $\begin{array}{l}\text { Industrial } \\
\text { production, } \\
\text { Credit } \\
\text { defaults all } \\
\text { sectors, } \\
\text { Wholesale, } \\
\text { and retail } \\
\text { trade }\end{array}$ & $\begin{array}{l}\text { Industrial } \\
\text { cycle affects } \\
\text { the sectoral } \\
\text { credit-default } \\
\text { cycles at } \\
\text { different. } \\
\text { time scales }\end{array}$ & - & $\begin{array}{l}\mathrm{MSE}= \\
0.00022\end{array}$ \\
\hline 5 & $\begin{array}{l}\text { Aigun et } \\
\text { al. }(2020)\end{array}$ & $\begin{array}{l}\text { Risk } \\
\text { assessmen } \\
\mathrm{t} \text { of } \\
\text { logistic } \\
\text { finance }\end{array}$ & $\begin{array}{l}\text { BPNN and } \\
\text { Fuzzy } \\
\text { mathemati } \\
\text { cal }\end{array}$ & 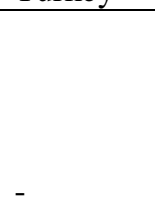 & 2019 & $x_{1}$ & $\begin{array}{l}\mathrm{NN}+\text { Fuzzy is } \\
\text { accurate in the } \\
\text { prediction }\end{array}$ & - & (1) \\
\hline 6 & $\begin{array}{l}\text { Baesens et } \\
\text { al. (2003) }\end{array}$ & $\begin{array}{l}\text { Rule } \\
\text { Extraction } \\
\text { and } \\
\text { Decision } \\
\text { Tables }\end{array}$ & $\begin{array}{l}\text { MLP, } \\
\text { Neuro } \\
\text { rule, } \\
\text { Trepan, } \\
\text { and Nef }\end{array}$ & $\begin{array}{l}\text { German } \\
\text { credit } \\
\text { dataset } \\
\text { from } \\
\text { UCI }\end{array}$ & - & $\begin{array}{l}\text { Term of } \\
\text { loan, } \\
\text { Purpose, } \\
\text { savings } \\
\text { account }\end{array}$ & $\begin{array}{l}\text { Extract very } \\
\text { compact rule } \\
\text { sets and trees } \\
\text { for all data } \\
\text { sets }\end{array}$ & - & - \\
\hline
\end{tabular}




\begin{tabular}{|c|c|c|c|c|c|c|c|c|c|}
\hline & & $\begin{array}{l}\text { for Credit- } \\
\text { Risk } \\
\text { Evaluatio } \\
\mathrm{n}\end{array}$ & class. & $\begin{array}{l}\text { repositor } \\
\mathrm{y}, \text { Bene1 } \\
\text { and } \\
\text { Bene2 } \\
\text { datasets } \\
\text { from } \\
\text { Benelue } \\
x \\
\text { financial } \\
\text { institutio } \\
\text { ns }\end{array}$ & & $\begin{array}{l}\text { balance, } \\
\text { income, } \\
\text { property, } \\
\text { No. of years } \\
\text { as a client, } \\
\text { Economical } \\
\text { sector }\end{array}$ & & & \\
\hline 7 & $\begin{array}{l}\text { Chikolwa } \\
\text { and Chan } \\
(2008)\end{array}$ & $\begin{array}{l}\text { Determina } \\
\text { nts of } \\
\text { credit } \\
\text { ratings }\end{array}$ & $\begin{array}{l}\text { Standard } \\
\text { NN Vs } \\
\text { Ordinal } \\
\text { regression }\end{array}$ & $\begin{array}{l}\text { MBS } \\
\text { credit } \\
\text { ratings } \\
\text { of } \\
\text { Standard } \\
\text { and Poor }\end{array}$ & $\begin{array}{l}1999- \\
2005\end{array}$ & $\begin{array}{l}\text { Loan to } \\
\text { value; Debt } \\
\text { Service } \\
\text { Coverage } \\
\text { Ratio; issue } \\
\text { size; bond } \\
\text { tenure, } \\
\text { property } \\
\text { diversity, } \\
\text { geographica } \\
\text { l diversity, } \\
\text { CMBS } \\
\text { rating }\end{array}$ & $\begin{array}{l}\text { ANN > } \\
\text { Ordinal } \\
\text { regression }\end{array}$ & $\begin{array}{l}\text { Pseudo R } \\
\text { squared = } \\
0.018\end{array}$ & $\begin{array}{l}\text { Classificati } \\
\text { on } \\
\text { accuracy = } \\
80 \%\end{array}$ \\
\hline 8 & $\begin{array}{l}\text { Trinkle } \\
\text { and } \\
\text { Baldwin } \\
(2007)\end{array}$ & $\begin{array}{l}\text { Interpreta } \\
\text { ble credit } \\
\text { model } \\
\text { developm } \\
\text { ent using } \\
\text { NN }\end{array}$ & $\begin{array}{l}\text { ANN } \\
\text { models } \\
\text { created } \\
\text { from } \\
\text { previous } \\
\text { research } \\
\text { studies }\end{array}$ & $\begin{array}{l}\text { Two } \\
\text { German } \\
\text { consume } \\
\text { r credit } \\
\text { data } \\
\text { sets, } \\
\text { SAS } \\
\text { data } \\
\text { repositor } \\
\text { y }\end{array}$ & 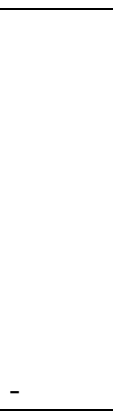 & $\begin{array}{l}\text { Age, car, } \\
\text { cards, Cash, } \\
\text { etc. }\end{array}$ & $\begin{array}{l}\text { ANN > } \\
\text { General credit } \\
\text { models }\end{array}$ & 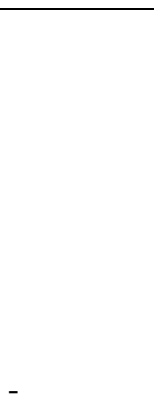 & $\begin{array}{l}\text { Accuracy } \\
\text { rate }= \\
0.63084\end{array}$ \\
\hline 9 & $\begin{array}{l}\text { Hajek } \\
(2011)\end{array}$ & $\begin{array}{l}\text { Municipal } \\
\text { credit } \\
\text { rating } \\
\text { modeling } \\
\text { by neural } \\
\text { networks }\end{array}$ & $\begin{array}{l}\text { FFNN, } \\
\text { RBFNN, } \\
\text { Probabilist } \\
\text { ic NN, } \\
\text { Cascade } \\
\text { correlation } \\
\text { NN, Group } \\
\text { method of } \\
\text { data } \\
\text { handling } \\
\text { (GMDH) } \\
\text { polynomia } \\
\text { 1 NNs, } \\
\text { Support } \\
\text { Vector } \\
\text { Machines. }\end{array}$ & $\begin{array}{l}\text { Credit } \\
\text { informat } \\
\text { ion's of } \\
\text { r } 169 \\
\text { US } \\
\text { municip } \\
\text { alities } \\
\text { (located } \\
\text { in the } \\
\text { State of } \\
\text { Connect } \\
\text { icut) }\end{array}$ & $\begin{array}{l}2003- \\
2007\end{array}$ & $\begin{array}{l}\text { Population. } \\
\text { Population } \\
\text { growth, } \\
\text { median } \\
\text { family } \\
\text { income, } \\
\text { unemploym } \\
\text { ent rate, } \\
\text { total } \\
\text { revenue to } \\
\text { total } \\
\text { expenditure, } \\
\text { tax revenue } \\
\text { to total } \\
\text { revenue, tax } \\
\text { collectibles, } \\
\text { debt } \\
\text { service, } \\
\text { total debt to } \\
\text { the total } \\
\text { population, } \\
\text { tax }\end{array}$ & $\begin{array}{l}\text { An accurate } \\
\text { credit rating } \\
\text { classification }\end{array}$ & & $\begin{array}{l} \\
\\
\text { PNN, } \\
\text { Classificati } \\
\text { on } \\
\text { Accuracy } \\
\text { test }= \\
98.8 \% \\
\end{array}$ \\
\hline
\end{tabular}




\begin{tabular}{|c|c|c|c|c|c|c|c|c|c|}
\hline & & & & & & $\begin{array}{l}\text { collection } \\
\text { rate, form } \\
\text { of income. }\end{array}$ & & & \\
\hline $\begin{array}{l}1 \\
0\end{array}$ & $\begin{array}{l}\text { Zan et al. } \\
(2004)\end{array}$ & $\begin{array}{l}\text { Credit } \\
\text { rating } \\
\text { analysis } \\
\text { with } \\
\text { support } \\
\text { vector } \\
\text { machines } \\
\text { and neural } \\
\text { networks }\end{array}$ & $\begin{array}{l}\text { backpropa } \\
\text { gation } \\
\text { neural } \\
\text { network } \\
\text { (BNN) Vs } \\
\text { Support } \\
\text { vector } \\
\text { machines }\end{array}$ & $\begin{array}{l}\text { Taiwan } \\
\text { Ratings } \\
\text { Corporat } \\
\text { ion, } \\
\text { Securitie } \\
\text { s and } \\
\text { Futures } \\
\text { Institute, } \\
\text { S\&P } \\
\text { Compus } \\
\text { tat data } \\
\text { set. }\end{array}$ & $\begin{array}{l}1991 \text { to } \\
2000\end{array}$ & $\begin{array}{l}\text { TA, TL, } \\
\text { DE, CR, } \\
\text { ROA, ROE, } \\
\text { EPS, NOI, } \\
\text { NII, etc. }\end{array}$ & $\begin{array}{l}\text { BNN, SVM } \\
\text { >Linear } \\
\text { regression }\end{array}$ & - & $\begin{array}{l}\text { Accuracy } \\
\text { rate }=80 \%\end{array}$ \\
\hline
\end{tabular}

\# Cross validation

\section{Financial Distress: \\ Optimized NN -}

Multi-layer perceptron (MLP) -Loukeris and Eleftheriadis (2015) used MLP, hybrid MLP with neurogenetic, and voted perceptron algorithm (VPA). VPA is a method that linearly separates data with a larger margin to predict financial distress. Manel (2012) used five MLP and compared them with traditional financial analysis to predict financial distress and found that the MLP is superior in accuracy.

Learning vector quantization ( $L V Q)$ - LVQ is a NN method with a supervised algorithm to let choose the number of training instances to hang on to. Brockett et al. (2006) compared the multiple discriminant analysis (MDA) and logistic regression with LVQ to analyze the solvency of life insurance companies and found $\mathrm{NN}$ architectures are superior in predictions.

Hidden layer learning vector quantization - Like LVQ, hidden layer LVQ (HDLVQ) outperforms the traditional NN methods and financial techniques while evaluating the corporate solvency of life insurance companies. The research study by Neves and Vieira (2006) integrated HDLVQ to correct the outputs of MLP and found the technique is superior to traditional techniques like z core models and standard NN.

\section{Hybrid NN -}

Fuzzy analytical hierarchy and CAMEL model - CAMELS framework is the most widely applied methodology to study the financial position of banks. Wanke et al. (2016) used NN with a fuzzy analytical hierarchical model along the CAMELS framework to predict the financial distress of banks.

$Z$ score model $-Z$ score model is a financial technique to evaluate the financial distress of a company. Pradhan (2011) used NN along with Z score and found it classifies accurately.

Profitability index and capital structure variables -Willer et al. (2020) created a business insolvency forecasting model using $\mathrm{NN}$ and found that the predictable power of NN shows significant accuracy. Yang et al. (1998) and Atiya (2001) confirms the accuracy. 
Table 5. NN literatures in Financial distress

\begin{tabular}{|c|c|c|c|c|c|c|c|c|c|}
\hline & Author(s) & Purpose & $\begin{array}{l}\text { NN } \\
\text { Model }\end{array}$ & Sample & $\begin{array}{l}\text { Output } \\
\text { Variable }\end{array}$ & Predictors & Comparison & $\begin{array}{l}\mathbf{R} \\
\text { Squared } \\
\text { Value }\end{array}$ & Accuracy \\
\hline 1 & $\begin{array}{l}\text { Loukeris } \\
\text { and } \\
\text { Eleftheria } \\
\text { dis }(2015)\end{array}$ & $\begin{array}{l}\text { Credit } \\
\text { portfolio } \\
\text { selection } \\
\text { process }\end{array}$ & $\begin{array}{l}\text { MLP, } \\
\text { hybrid } \\
\text { MLP } \\
\text { with } \\
\text { neurog } \\
\text { enetic, } \\
\text { and } \\
\text { voted } \\
\text { percept } \\
\text { ron } \\
\text { algorith } \\
\text { m } \\
\end{array}$ & $\begin{array}{l}1411 \\
\text { compan } \\
\text { ies from } \\
\text { Greek } \\
\text { commer } \\
\text { cial } \\
\text { bank }\end{array}$ & $\begin{array}{l}1994 \text { to } \\
1997\end{array}$ & $\begin{array}{l}16 \text { Financial } \\
\text { ratios }\end{array}$ & (2) & 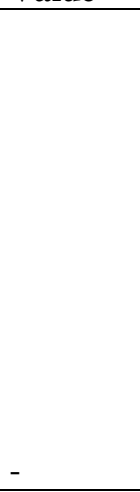 & MSE 0.034 \\
\hline 2 & $\begin{array}{l}\text { Manel } \\
(2012)\end{array}$ & $\begin{array}{l}\text { Predictio } \\
\mathrm{n} \text { of } \\
\text { financial } \\
\text { distress }\end{array}$ & BPNN & $\begin{array}{l}528 \\
\text { Tunisia } \\
\mathrm{n} \text { firm } \\
\text { (Central } \\
\text { bank of } \\
\text { Tunisia } \\
\text { report) }\end{array}$ & $1999-2006$ & $\begin{array}{l}26 \text { financial } \\
\text { ratios predicting } \\
\text { distress }\end{array}$ & - & - & $\begin{array}{l}\text { Classificati } \\
\text { on } \\
\text { accuracy - } \\
98.9 \%\end{array}$ \\
\hline 3 & $\begin{array}{l}\text { Brockett } \\
\text { et al. } \\
(2006)\end{array}$ & $\begin{array}{l}\text { Compari } \\
\text { son of } \\
\text { NN and } \\
\text { statistical } \\
\text { models } \\
\text { for life } \\
\text { insurers' } \\
\text { financial } \\
\text { distress } \\
\text { predictio } \\
\text { n }\end{array}$ & $\begin{array}{l}\text { back- } \\
\text { propag } \\
\text { ation } \\
\text { and } \\
\text { learnin } \\
\mathrm{g} \\
\text { vector } \\
\text { quantiz } \\
\text { ation } \\
\text { (LVQ) } \\
\text { Vs } \\
\text { multipl } \\
\text { e } \\
\text { discrim } \\
\text { inant } \\
\text { analysi } \\
\text { s and } \\
\text { logistic } \\
\text { regressi } \\
\text { on } \\
\text { analysi } \\
\text { s } \\
\end{array}$ & $\begin{array}{l}\text { Texas } \\
\text { Depart } \\
\text { ment of } \\
\text { Insuran } \\
\text { ce data } \\
\end{array}$ & $\begin{array}{l}1991 \text { to } \\
1995\end{array}$ & 更 & $\begin{array}{l}\text { BNN, LVQ > } \\
\text { MDA, } \\
\text { Logistic } \\
\text { Regression }\end{array}$ & - & $\begin{array}{l} \\
\\
\text { Correct rate } \\
(1994)= \\
\text { LVQ } \\
(1.00), \text { BP } \\
(0.971)\end{array}$ \\
\hline 4 & $\begin{array}{l}\text { Neves and } \\
\text { Vieira } \\
(2006) \#\end{array}$ & $\begin{array}{l}\text { Improvin } \\
\mathrm{g} \\
\text { Bankrupt } \\
\text { cy } \\
\text { Predictio } \\
\mathrm{n} \\
\text { with } \\
\text { Hidden } \\
\text { Layer }\end{array}$ & $\begin{array}{l}\text { Learnin } \\
\text { g } \\
\text { vector } \\
\text { quantiz } \\
\text { ation + } \\
\text { Multi- } \\
\text { layer } \\
\text { percept } \\
\text { ron }\end{array}$ & $\begin{array}{l}780,000 \\
\text { financia } \\
1 \\
\text { stateme } \\
\text { nts of } \\
\text { French } \\
\text { compan } \\
\text { ies, } \\
\text { Industri }\end{array}$ & $1998-2000$ & $\begin{array}{l}\text { Input consists of } \\
30 \text { financial } \\
\text { ratios }\end{array}$ & & & $\begin{array}{l}\text { Generalisat } \\
\text { ion error: } \\
\text { MLP = } \\
8.8 \% \text {, } \\
\text { HLVC-Q = } \\
7.3 \%\end{array}$ \\
\hline
\end{tabular}




\begin{tabular}{|c|c|c|c|c|c|c|c|c|c|}
\hline & & $\begin{array}{l}\text { Learning } \\
\text { Vector } \\
\text { Quantiza } \\
\text { tion }\end{array}$ & & $\begin{array}{l}\text { al } \\
\text { French } \\
\text { firms, } \\
583 \\
\text { bankrup } \\
\text { t firms } \\
\end{array}$ & & & & & \\
\hline 5 & $\begin{array}{l}\text { Wanke et } \\
\text { al. (2016) }\end{array}$ & $\begin{array}{l}\text { Predictin } \\
\text { g } \\
\text { performa } \\
\text { nce in } \\
\text { ASEAN } \\
\text { banks }\end{array}$ & $\begin{array}{l}\text { Fuzzy } \\
\text { analytic } \\
\text { hierarc } \\
\text { hy } \\
\text { process } \\
\text { NN }\end{array}$ & $\begin{array}{l}\text { Financi } \\
\text { al ratios } \\
\text { of } 88 \\
\text { Associa } \\
\text { tion of } \\
\text { Southea } \\
\text { st Asian } \\
\text { Nations } \\
\text { bank } \\
\end{array}$ & $\begin{array}{l}2010 \text { to } \\
2013\end{array}$ & CAMELS ratios & $\begin{array}{l}\text { Possible to } \\
\text { explain the } \\
\text { causes of } \\
\text { inefficiency } \\
\text { using NN }\end{array}$ & - & $\begin{array}{l}\text { RMSE = } \\
0.0248\end{array}$ \\
\hline 6 & $\begin{array}{l}\text { Pradhan } \\
(2011)\end{array}$ & $\begin{array}{l}\text { Predictio } \\
\mathrm{n} \text { of } \\
\text { financial } \\
\text { distress }\end{array}$ & BPNN & $\begin{array}{l}\text { State } \\
\text { Bank of } \\
\text { India }\end{array}$ & $2001-2010$ & $Z$ score variables & - & - & - \\
\hline 7 & $\begin{array}{l}\text { Willer et } \\
\text { al. }(2020)\end{array}$ & $\begin{array}{l}\text { Forecasti } \\
\text { ng } \\
\text { business } \\
\text { insolvenc } \\
\mathrm{y}\end{array}$ & $\begin{array}{l}\text { Standar } \\
\text { d NN }\end{array}$ & - & 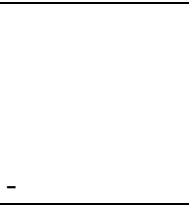 & $\begin{array}{l}\text { Profitability } \\
\text { index, capital } \\
\text { structure }\end{array}$ & - & - & $\begin{array}{l}91 \% \\
\text { Accuracy } \\
\text { Dynamic } \\
\text { Model }\end{array}$ \\
\hline 8 & $\begin{array}{l}\text { Yang et } \\
\text { al. (1998) }\end{array}$ & $\begin{array}{l}\text { Probabili } \\
\text { stic } \\
\text { Neural } \\
\text { Network } \\
\text { s in } \\
\text { Bankrupt } \\
\text { cy } \\
\text { Predictio } \\
\text { n } \\
\end{array}$ & $\begin{array}{l}\text { Fisher } \\
\text { discrim } \\
\text { inant } \\
\text { analysi } \\
\text { s, } \\
\text { BPNN, } \\
\text { PNN, } \\
\text { PNN } \\
\text { without } \\
\text { patterns } \\
\text { normali } \\
\text { zed. }\end{array}$ & $\begin{array}{l}122 \\
\text { compan } \\
\text { ies U.S. } \\
\text { oil and } \\
\text { gas } \\
\text { industry }\end{array}$ & $\begin{array}{l}1984 \text { to } \\
1989\end{array}$ & $\begin{array}{l}\text { Net cash flow to } \\
\text { total assets, } \\
\text { TD/TA, CL/TD, } \\
\text { etc }\end{array}$ & $\begin{array}{l}\text { Fischer } \\
\text { discriminant } \\
\text { analysis, } \\
\text { PNN } \\
\text { normalized> } \\
\text { BP NN, } \\
\text { Probabilistic } \\
\text { NN without a } \\
\text { pattern }\end{array}$ & - & $\begin{array}{l}\text { Fisher } \\
\text { discriminan } \\
\mathrm{t} \text { analysis = } \\
87 \% \\
\text { correct } \\
\text { classificati } \\
\text { on, }\end{array}$ \\
\hline 9 & $\begin{array}{l}\text { Atiya } \\
(2001)\end{array}$ & $\begin{array}{l}\text { Bankrupt } \\
\text { cy } \\
\text { Predictio } \\
\text { n for } \\
\text { Credit } \\
\text { Risk }\end{array}$ & $\begin{array}{l}\text { Standar } \\
\text { d NN }\end{array}$ & $\begin{array}{l}\text { Defaulte } \\
\mathrm{d} \text { and } \\
\text { from } \\
\text { solvent } \\
\text { US } \\
\text { firms, } \\
716 \\
\text { solvent } \\
\text { firms } \\
\text { and } 195 \\
\text { defaulted } \\
\text { firm }\end{array}$ & - & $\begin{array}{l}\text { Merton's asset- } \\
\text { based model }\end{array}$ & - & - & $\begin{array}{l}\text { Correct rate } \\
=85.50 \%\end{array}$ \\
\hline
\end{tabular}

\# Cross validation

\section{Other Financial Applications:}

Standard NN

Apart from the major topics of research, researchers have found $\mathrm{NN}$ is efficient to detect fraudulent reporting (Koskivaara \& Back, 2007; Omar et al. 2017) and project portfolio management (Costantino et al., 2015). 


\section{Optimized NN -}

A fuzzy analytical model is used for creating a financial information system (Wang et al., 2020).

A self-organization map algorithm, an unsupervised learning NN that produces a lowdimensional, discretized representation of the input space of the training samples, is called a map. SOM is used to analyze the integration of EU capital markets (Horobet 2014), differences in world economies (Cimpoeru 2015).

\section{Hybrid NN -}

NN with the Ohlson model is used to predict operating performance (Ying-Hua \& Shih-Chin, 2013).

\section{CONCLUSION}

A descriptive systematic review was conducted to find the application of neural networks in financial research. The study found a keen research interest to use NN for predicting financial data. This is obvious from the statistic that about $53 \%$ of the collected research studies applied $\mathrm{NN}$ in investment prediction. Credit evaluation and financial distress topics contribute to $20 \%$ and $17 \%$ of each of the collected papers. There are very few works (10\%) found on other financial aspects.

The following are the reflections of the review. First, it is observed that the researchers have used arbitrary data partition and architecture selection in all the research works. Besides, the performance or evaluation metrics widely differ among the collected research studies, giving less scope for comparing the accuracy of an NN architecture in a particular subtopic. Hence, in this study, a meta-analytic comparison to generalize the NN architectures and formalizing a theory to choose a suitable NN method under a topic has serious limitations.

Second, there are few studies (Neves \& Vieira, 2006; Qi \& Zhao, 2011) that have performed the cross-validation in NN models. Cross-validation increases efficiency in using financial data as every observation is used for both training and testing which results in a more accurate estimate of out-of-sample prediction. Further, overfitting and underfitting of data will be managed efficiently through cross-validation.

Third, unlike prediction, in the research studies of classifying financial data, there is a scope for a meta-analysis based on generalizing Area Under Curve (AUC) that help to estimate the accuracy of classification on a particular topic. Further, the study observed NN architectures including domain-specific knowledge performs with more accuracy. Hence, more domain-based Hybrid NN architectures can be trained.

Besides, the review has the following limitations. The descriptive systematic review has examined only the research papers published and available under the EBSCO database. Consequently, the works of the literature review are prone to publication bias, which occurs with publishing only statistically significant results. Beyond, the research works in conference proceedings and working papers are not reviewed. Hence some sub-topics and main research topics might have been remaining uncovered. Systematically including more research papers from other sources will improve the chance of a meta-analysis of NN in financial research. Since meta-analysis on machine learning by Krittanawong et al. (2020) and Roelofs et al. (2019) are the only works available, that too in the medical domain, a meta-analysis of NN architectures in financial research will be a significant contribution to the existing financial and NN literature. 


\section{REFERENCES}

Altman, E., Marco, G., \& Varetto, F. (1994). Corporate Distress Diagnosis: Comparisons Using Linear Discriminant Analysis and Neural Networks (the Italian Experience). Journal of Banking and Finance, 18(3), 505-529.

Aiqun, W., Zicong, H., Yilin, W., Kolivand, H., Balas, V. E., Paul, A., \& Ramachandran, V. (2020). Risk assessment of logistics finance enterprises based on BP neural network and fuzzy mathematical model. Journal of Intelligent \& Fuzzy Systems, 39(4), 5915-5925. https://doi.org/10.3233/JIFS-189066

Atiya, A.F. (2001). Bankruptcy prediction for credit risk using neural networks: A survey and new results, IEEE Transactions on Neural Networks, 12(1), 929-935.

Baesens, B., Setiono, R., Mues, C., \& Vanthienen, J. (2003). Using Neural Network Rule Extraction and Decision Tables for Credit-Risk Evaluation. Management Science, 49(3), 312-329. https://doi.org/10.1287/mnsc.49.3.312.12739

Barr, D., \& Mani. G. (1994). Using neural nets to manage investments, AI EXPERT, 34(3), 1622.

Barnes, B, M., \& Lee, C, S, V. (2009). Effects of Macroeconomic and Firm-Specific Factors on Shareholder Wealth: Some Australian Evidence. The Journal of wealth management, 12 (1), 41-61. https://doi.org/10.3905/jwm.2009.12.1.041

Brockett, P. L., Golden, L. L., Jang, J., \& Yang, C. (2006). A Comparison of Neural Network, Statistical Methods, and Variable Choice for Life Insurers' Financial Distress Prediction. Journal of Risk \& Insurance, 73(3), 397-419. https://doi.org/10.1111/j.15396975.2006.00181.x

Brooks, C., Andreas, G, F., Hoepner, McMillan, D., Vivian, A, \& Simen, C, W. (2019) Financial data science: the birth of a new financial research paradigm complementing econometrics? The European Journal of Finance, 25(17), 1627-1636, https://doi.org/10.1080/1351847X.2019.1662822

Blynski, L., \& Faseruk, A. (2006). Comparison of the effectiveness of option price forecasting: Black-Scholes vs. simple and hybrid neural networks. Journal of Financial Management \& Analysis, 19(2), 46.

Cavalcantea, R, C., Brailerio, C, R., Souza, L, V., Nobrega, P, J., Oliveria, L, A. (2016). Computational Intelligence and financial markets: A survey and future directions. Expert systems with applications, 55, 194 - 211. http://dx.doi.org/10.1016/j.eswa.2016.02.006

Chang, P. C., Liu, C. H., Fan, C. Y., Lin, J. L., \& Lai, C. M. (2009). An Ensemble of Neural Networks for Stock Trading Decision Making. In: Huang DS., Jo KH., Lee HH., Kang HJ., Bevilacqua V. (eds) Emerging Intelligent Computing Technology and Applications. With Aspects of Artificial Intelligence. ICIC 2009. Lecture Notes in Computer Science, 5755. https://doi.org/10.1007/978-3-642-04020-7_1 
Chen, F., \& Sutcliffe, C. (2012). Pricing and Hedging Short Sterling Options Using Neural Networks. Intelligent Systems in Accounting, Finance \& Management, 19(2), 128-149. https://doi.org/10.1002/isaf.336

Chikolwa, B., \& Chan, F. (2008). Determinants of Commercial Mortgage-Backed Securities Credit Ratings: Australian Evidence. International Journal of Strategic Property Management, 12(2), 69-94. https://doi.org/10.3846/1648-715X.2008.12.69-94

Chiang, W.C., Urban, T. L. \& Baldridge, G.W. (1996). A neural network approach to mutual fund net asset value forecasting, Omega - International journal of management science, 24, 205-215.

Cifter, A., Yilmazer, S., \& Cifter, E. (2009). Analysis of sectoral credit default cycle dependency with wavelet networks: Evidence from Turkey. Economic Modelling, 26(6), 1382-1388. https://doi.org/10.1016/j.econmod.2009.07.014

Cimpoeru, S. (2017). Using Self organizing maps for assessing systemic risk. Evidences form global economic crisis. The Bucharest University of Economic Studies. http://www.ecocyb.ase.ro/nr20152/05\%20-\%20Cimpoeru_Smaranda.pdf

Coakley, J., \& Brown. C. (2000). Artificial neural networks in accounting and finance: Modelling issues. Intelligent Systems in Accounting Finance \& Management,9(2).

Costnatino, F., Gravio, G, D., \& Nonino, F. (2015). Project selection in project portfolio management: An artificial neural network model based on critical success factors. International journal of project management, 33,1744 - 1754. http://dx.doi.org/10.1016/j.ijproman.2015.07.003

Dunis, C. L., Laws, J., Middleton, P. W., \& Karathanasopoulos, A. (2015). Trading and hedging the corn/ethanol crush spread using time-varying leverage and nonlinear models. $\begin{array}{llll}\text { European of } \quad \text { Journal 352-375. } & \text { 21(4), }\end{array}$ https://doi.org/10.1080/1351847X.2013.830140

Fadlalla, A., \& Lin, C. (2001) An analysis of the applications of neural networks in finance. Interfaces, 31(4), 112-122.

Feldman, K, \& Kingdon, J. (1995). Neural networks and some applications to finance, Applied Mathematical Finance, 2(1), 17-42. https://doi.org/10.1080/13504869500000002

Gençay, R., \& Gibson, R. (2007). Model Risk for European-Style Stock Index Options. IEEE Transactions on Neural Networks, 18(1), 193-202. https://doi.org/10.1109/TNN.2006.883005

Gradojevic, N., Gençay, R., \& Kukolj, D. (2009). Option Pricing With Modular Neural Networks. IEEE Transactions on Neural Networks, 20(4), 626-637. https://doi.org/10.1109/TNN.2008.2011130 
Gupta, P., Chauhan, S. \& Jaiswal, M.P. (2019). Classification of Smart City Research - a Descriptive Literature Review and Future Research Agenda. Information System Frontiers, 21, 661-685. https://doi.org/10.1007/s10796-019-09911-3

Gustafson, J., L. (2011). Moore's Law. In: Padua D. Encyclopedia of Parallel Computing. Springer, Boston, MA. https://doi.org/10.1007/978-0-387-09766-4_81

Hutchison A.W, Lo A.W, \& Poggio, T. (1994) A nonparametric approach to pricing and hedging derivative securities via learning networks. The journal of Finance, 49(3), 851889.

Hajek, P. (2011). Municipal credit rating modelling by neural networks. Decision support systems, 51 (1), 108 - 118. https://doi.org/10.1016/j.dss.2010.11.033

Hariri, R. H., Fredericks, E.M. \& Bowers, K. M. (2019). Uncertainty in big data analytics: survey, opportunities, and challenges. Journal of Big Data,6. https://doi.org/10.1186/s40537-019-0206-3.

Haefke, C., \& Helmenstein, C. (2002). Index forecasting and model selection. International Journal of Intelligent Systems in Accounting Finance \& Management, 11(2), 119-135. https://doi.org/10.1002/isaf.214

Horobet, A., Belascu, L., Ionita, I., \& Serban - Oprescu, A.-T. (2014). A Neural Networks Perspective on the Financial integration of European Capital Markets. Economic Computation \& Economic Cybernetics Studies \& Research, 48(1), 1-12

Huang, G., Huang, G, B., Shiji, S., \& Youa, K. (2014). Trends in extreme learning machines: A review. Neural Networks. 61, 34 - 45. http://dx.doi.org/10.1016/j.neunet.2014.10.001

Huang, J., Chai, J. \& Cho, S. (2020). Deep learning in finance and banking: A literature review and classification. Frontiersof Business Research in China, 14. https://doi.org/10.1186/s11782-020-00082-6

Indro, D. C., Jiang, C. X., Patuwo, B. E., \& Zhang, G. P. (1999). Predicting mutual fund performance using artificial neural networks. Omega, 27(3), 373-380. https://doi.org/10.1016/S0305-0483(98)00048-6

Jan, M. N., \& Ayub, U. (2019). Do the Fama and French Five-Factor Model Forecast Well Using Ann? Journal of Business Economics \& Management, 20(1), 168-191. https://doi.org/10.3846/jbem.2019.8250

Kaastra, I. \& Boyd, M. (1996). Designing a Neural Network for Forecasting Financial and Economic Time Series. Neurocomputing, 10, 215-236. https://doi.org/10.1016/09252312(95)00039-9 
Karathanasopoulos, A., Dunis, C., \& Khalil, S. (2016). Modeling, forecasting, and trading with a new sliding window approach: the crack spread example. Quantitative Finance, 16(12), 1875-1886. https://doi.org/10.1080/14697688.2016.1211796

Karunananthan, S., Maxwell, L, J., \& Welch, V. (2020) Protocol: When and how to replicate systematic reviews. Campbell Systematic Reviews. 16. https://doi.org/10.1002/cl2.1087

Krishnaswamy, C.R., Gilbert, E.W., \& Pashley, M.M. (2000) Neural network applications in finance: A practical introduction. Financial Practice and Education, 10(1), 75-84.

Kriteenawong, C., Virk, H, H., Bangalore, S., Wang, Z., Johnson, W, K., Rachel. (2020). Machine learning prediction in cardiovascular diseases: a meta-analysis. Scientific reports, 10. https://doi.org/10.1038/s41598-020-72685-1

Kohler, M., Krzyżak, A., \& Todorovic, N. (2010). Pricing of High-Dimensional American Options by Neural Networks. Mathematical Finance, 20(3), 383-410. https://doi.org/10.1111/j.1467-9965.2010.00404.x

Koskivaara, E., \& Back, B. (2007). Artificial Neural Network Assistant (ANNA) for Continuous Auditing and Monitoring of Financial Data. Journal of Emerging Technologies in Accounting, 4, 29-45. https://doi.org/10.2308/jeta.2007.4.1.29

Kim, K. (2004). Artificial neural networks with feature transformation based on domain knowledge for the prediction of stock index futures. Intelligent Systems in Accounting, Finance \& Management, 12(3), 167-176. https://doi.org/10.1002/isaf.252

Light, R.J., \& Pillemer, D. B. (1984). Summing up: The science of reviewing research. Cambridge, MA: Harvard University Press.

Lin, C.-T., \& Yeh, H.-Y. (2009). Empirical of the Taiwan stock index option price forecasting model - applied artificial neural network. Applied Economics, 41(15), 1965-1972. https://doi.org/10.1080/00036840601131672

Loterman, G., Brown, I., Martens, D., Mues, C., \& Baesens, B. (2012). Benchmarking regression algorithms for loss given default modeling. International Journal of Forecasting, 28(1), 161-170. https://doi.org/10.1016/j.ijforecast.2011.01.006

Loukeris, N., \& Eleftheriadis, I. (2015). Further Higher Moments in Portfolio Selection and A Priori Detection of Bankruptcy, Under Multi-layer Perceptron Neural Networks, Hybrid Neuro-genetic MLPs, and the Voted Perceptron. International Journal of Finance \& Economics, 20(4), 341-361. https://doi.org/10.1002/ijfe.1521

LV, D., Wang, D., Li, M., \& Xiang, Y. (2020). DNN models based on dimensionality reduction for stock trading. Intelligent data analysis, 24(1), 19-45. https://doi.org/10.3233/IDA184403 
Manel, H. (2012). Prediction of Financial Distress for Tunisian Firms: A Comparative Study between Financial Analysis and Neuronal Analysis. Business Intelligence Journal, 5(2), 374-382.

Moher, D., Liberati, A., Tetzlaff, J., Altman, D, G., \& The PRISMA Group. (2009). Preferred Reporting Items for Systematic Reviews and Meta-Analyses: The PRISMA Statement. PLoS Med. 6(7). https://doi.org/10.1371/journal.pmed1000097

Moreno, D., \& Olmeda, I. (2007). Is the predictability of emerging and developed stock markets really exploitable? European Journal of Operational Research, 182(1), 436-454. https://doi.org/10.1016/j.ejor.2006.07.032

Ngai, E. W. T., \& Wat, F. K. T. (2002) A Literature Review and Classification of Electronic Commerce Research. Information \& Management, 39, 415-429.

Neves, J. C., \& Vieira, A. (2006). Improving bankruptcy prediction with Hidden Layer Learning Vector Quantization. European Accounting Review, 15(2), 253-271. https://doi.org/10.1080/09638180600555016

Omar, N., Johari, Z, A., \& Smith, M. (2017). Predicting fraudulent financial reporting using artificial neural network. Journal of Financial Crime, 24(2), 362-387. https://doi.org/10.1108/JFC-11-2015-0061

Ozbey, F., \& Paksoy, S. (2020). Estimation of the XU100 Index Return Volatility with the Integration of GARCH Family Models and ANN. Business and economics research journal, 11(2), $385-396$.

Petter, S., McLean, E. R. (2009). A meta-analytic assessment of the DeLone and McLean IS success model: An examination of IS success at the individual level. Information Management, 46(3), 159-166.

Pradhan, R., Pathak, K. K., \& Singh, V. P. (2011). Z Score Reveals Credit Capacity: A Case Study of SBI. International Journal of Financial Management, 1(3), 72-78.

Qi, M., \& Zhao, X. (2011). Comparison of modeling methods for Loss Given Default. Journal $\begin{array}{lll}\text { of banking } \quad \text { and } & \text { 2842-2855. }\end{array}$ https://doi.org/10.1016/j.jbankfin.2011.03.011

Roelofs, R., Shankar, V., Recht, B., Fridovich-Keil, S., Hardt, M., Miller, J \& Schmidt, L. (2019). A Meta-Analysis of Overfitting in Machine Learning. Advances in Neural Information Processing Systems 32 (Neur IPS 2019), 1-11. Retrieved from https://openreview.net/pdf/98fdb4bb89fd868a5dda9f827133eec6e11bb4fa.pdf

Safer, A. M. (2002). The application of neural networks to predict abnormal stock returns using insider trading data. Applied Stochastic Models in Business \& Industry, 18(4), 381-389. https://doi.org/10.1002/asmb.466 
Sapna, O., Botti, V., \& Argente, E. (2003). Application of neural networks to stock prediction in "pool” companies. Application of Artificial Intelligence 17(7):661-673.

Sariev, E., \& Germano, G. (2020) Bayesian regularized artificial neural networks for the estimation of the probability of default. Quantitative Finance, 20(2), 311-328. https://doi.org/10.1080/14697688.2019.1633014

Sermpinis, G., Laws, J., \& Dunis, C. L. (2013). Modeling and trading the realized volatility of the FTSE100 futures with higher-order neural networks. European Journal of Finance, 19(3), 165-179. https://doi.org/10.1080/1351847X.2011.606990

Shokraneh, F., \& Adams, C. E. (2019). Study-based registers reduce waste in systematic reviewing: discussion and case report. Systematic Reviews, 8(1), 129.

Sperckelsen, C., Mettenheim, H., \& Breitner, M. H. (2014). Real-Time Pricing and Hedging of Options on Currency Futures with Artificial Neural Networks. Journal of Forecasting, 33(6), 419-432. https://doi.org/10.1002/for.2311

Trinkle, B. S., \& Baldwin, A. A. (2007). Interpretable credit model development via artificial neural networks. Intelligent Systems in Accounting, Finance \& Management, 15(3/4), 123-147. https://doi.org/10.1002/isaf.289

Vellido A., Lisboa, P. J., Vaughan, J. (1999). Neural networks in business: a survey of applications (1992-1998). Expert Syst Appl. 17(1), 51-70.

Wie Zhang, A., Qing Cao, \& Schniederjans, M. J. (2004). Neural Network Earnings Per Share Forecasting Models: A Comparative Analysis of Alternative Methods. Decision Sciences, 35(2), 205-237. https://doi.org/10.1111/j.00117315.2004.02674.X

Wanke, P., Kalam Azad, M. A., Barros, C. P., \& Hadi, V. A. (2016). Predicting performance in ASEAN banks: an integrated fuzzy MCDM-neural network approach. Expert Systems, 33(3), 213-229. https://doi.org/10.1111/exsy.12144

Wang, A., Liu, Y., Isaeva, E., \& Rocha, Á. (2020). Intelligent financial management of company based on neural network and fuzzy volatility evaluation. Journal of Intelligent \& Fuzzy Systems, 38(6), 7215-7228. https://doi.org/10.3233/JIFS-179798

Wei, K., Nakamori, Y., Wang, S., \& Yu, L. (2007). Neural Networks in Finance and Economics Forecasting. International Journal of Information Technology \&Decision Making, 6(1), 113-140. https://doi.org/10.1142/S021962200700237X

Willer P.J., Giarola, V, A., Cardoso, C, A., \& Pinheiro, N., T. (2020). Business Insolvency Forecasting Using Artificial Neural Networks. Management and Development, 17(2), 136-162. https://doi.org/10.25112/rgd.v17i2.1777 
Wong, B. K., \& Selvi, Y. (1998). Neural network applications in finance: A review and analysis of literature (1990-1996). Information and Management, 34(3), 129-139.

Yang, Z. R., Platt, M. B., Platt, H. D (1999). Probabilistic neural networks in bankruptcy prediction. Journal of Business Research, 44(2), 67-74.

Ying-Hua, C., \& Shih-Chin, W. (2013). Integration of Evolutionary Computing and Equity Valuation Models to Forecast Stock Values Based on Data Mining. Asia Pacific Management Review, 18(1), 63-78. https://doi.org/10.6126/APMR.2013.18.1.04

Zan, H., Chen, H., Hsu, C., Chen, W., \& Wu, S. (2004). Credit rating analysis with support vector machines and neural networks: a market comparative study. Decision Support Systems, 37(4), 543-558. https://doi.org/10.1016/s0167-9236(03)00086-1

Zapart, C. A. (2003). Beyond Black-Scholes: A Neural Networks-Based Approach to Options Pricing. International Journal of Theoretical \& Applied Finance, 6(5), 469. https://doi.org/10.1142/S0219024903002006

\section{APPENDICES}

Appendix A. Figure depicting NN architectures in Investment Prediction

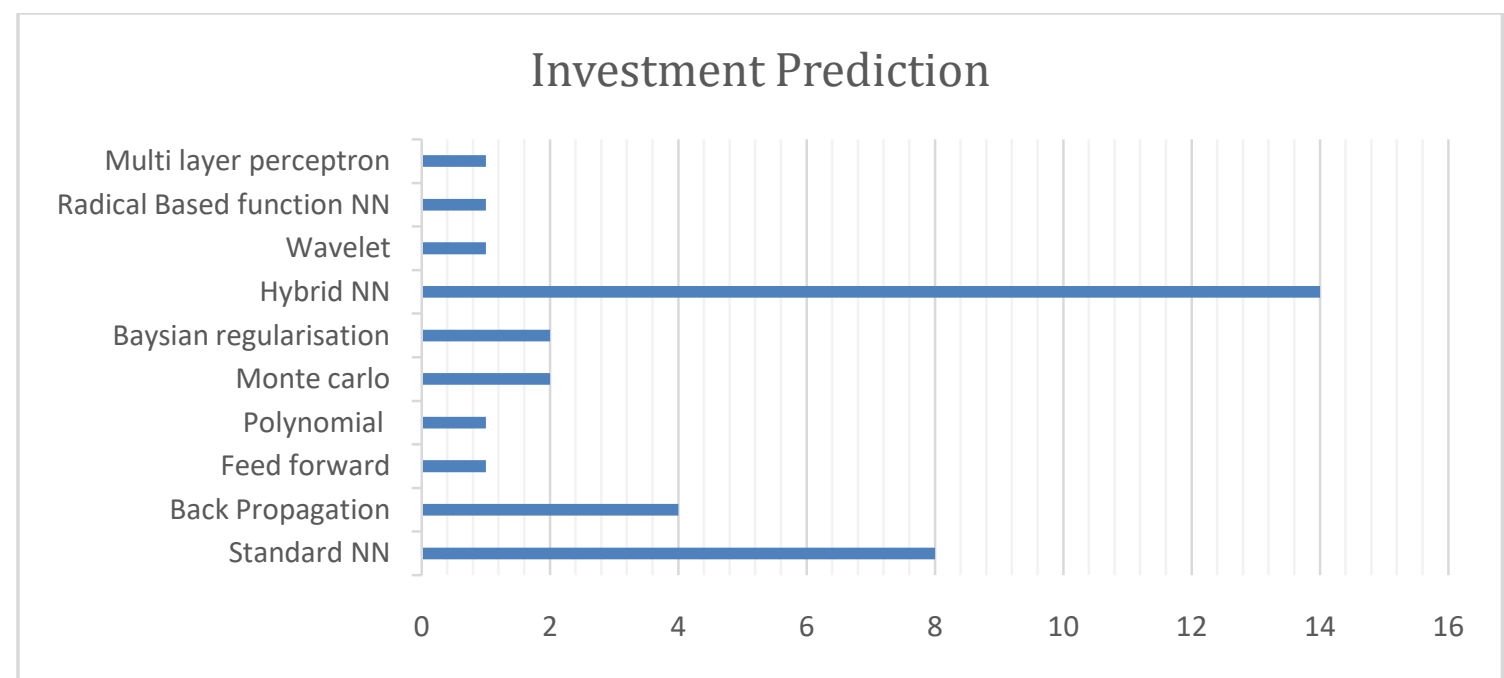

*Hybrid NN methods are developed by incorporating the financial theories (Black Scholes option pricing, Fama French five-factor model), advanced statistics (GARCH, PCA, LASSO, CART, PLR), advanced NN architectures (HONN, GPA, MLP), and domain-specific factors. 
Appendix B. Figure depicting NN architectures in credit evaluation, financial distress, and other applications

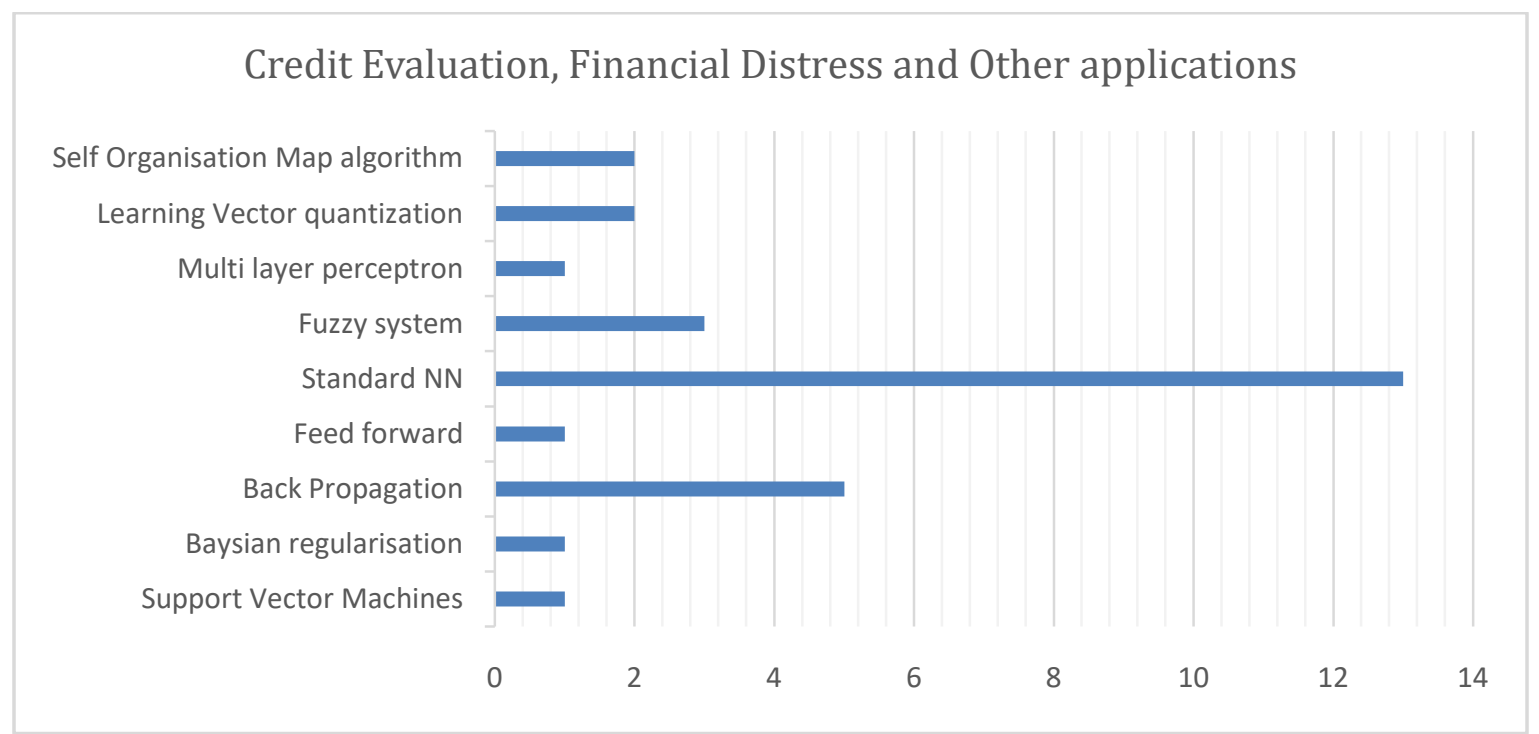

*Support Vector Machine is a similar machine learning algorithm like NN.

\section{Copyrights}

Copyright for this article is retained by the author(s), with first publication rights granted to the journal. This is an open-access article distributed under the terms and conditions of the Creative Commons Attribution license (http://creativecommons.org/licenses/by/4.0/) 